Cómo citar este trabajo: Merinero Rodríguez, R., Ledesma González, O. (2019). Analizando la gobernanza urbana mediante la aplicación del análisis de redes sociales (ARS): el caso del segundo plan estratégico de Málaga. Boletín de la Asociación de Geógrafos Españoles, 80, 2593, 1-38. http://dx.doi.org/10.21138/bage.2593

\title{
Analizando la gobernanza urbana mediante la aplicación del análisis de redes sociales (ARS): el caso del Segundo Plan Estratégico de Málaga
}

\author{
Analyzing urban governance through the application of social network \\ analysis (SNA): the case of the Second Strategic Plan of Malaga
}

\author{
Rafael Merinero Rodríguez \\ rmerrod@upo.es \\ Departamento de Sociología \\ Universidad Pablo de Olavide (España)
}

\section{Oswaldo Ledesma González \\ oledesma@ull.edu.es \\ Departamento de Geografía e Historia \\ Universidad de La Laguna (España)}

\section{Resumen}

La gobernanza entendida como una red de actores se ha convertido en el elemento central que caracteriza la elaboración e implementación de políticas públicas y se han identificado multitud de instrumentos que permiten su aplicación entre los que está la Planificación Estratégica Urbana. En este trabajo se propone un análisis del sistema de gobernanza del Segundo Plan Estratégico de Málaga mediante la aplicación del Análisis de Redes Sociales (ARS) que hace posible una caracterización estructural de la gobernanza y su vinculación con el nivel de ejecución de las políticas públicas.

Palabras clave: gobernanza; políticas públicas; planificación estratégica urbana; Análisis de Redes sociales (ARS); ciudad de Málaga. 


\begin{abstract}
Governance understood as a network of actors has become the central element characterizing the elaboration and implementation of public policies and a multitude of instruments have been identified allowing its application, among which is Urban Strategic Planning. This paper proposes an analysis of the governance system of the Second Strategic Plan of Malaga through the application of Social Network Analysis (ARS), enabling a structural characterization of governance and its link with the level of execution of public policies.
\end{abstract}

Key words: governance; public policies; urban strategic planning; Social Networks Analysis (SNA); city of Malaga.

\title{
1 Introducción
}

La gobernanza se refiere a los cambios en la acción pública emprendida por los gobiernos en un contexto cada vez más complejo y dinámico como consecuencia de una serie de procesos que favorecen un aumento de las interacciones y de las interdependencias entre problemas, territorios y actores (Le Galès, 2006). Entendida de esta manera, el elemento central de la misma lo constituye la red de relaciones entre actores públicos y privados para solucionar problemas públicos complejos. Por tanto, la colaboración y la cooperación entre estos actores en un ámbito concreto de política pública constituye el elemento fundamental de su adecuado diseño e implementación (Busetti \& Dente, 2018). Desde esta perspectiva, la gobernanza se concibe como una forma de gobierno que tiene como finalidad dar respuesta a la complejidad propia de la resolución de problemas públicos que requieren de la implicación y participación de un amplio número de actores públicos y privados. El elemento clave lo constituye las características de las redes de colaboración inter-actor, y su conocimiento y comprensión se convierten en el centro de atención no sólo para caracterizar la gobernanza como forma de gobierno, sino para descubrir los patrones de interacción y poder intervenir sobre ellos con el objetivo de incidir en la mejora la acción pública (Barzelay, 2007; O’Toole, 2015; Warsen, Nederhand, Klijn, Grotenberg \& Koppenjan, 2018).

De una manera más específica, la gobernanza como forma de gobierno ha venido siendo destacada como el componente definitorio de las políticas urbanas en las que las redes de colaboración y participación de actores públicos y privados tienen un destacado valor analítico (Blanco, 2013). La literatura científica sobre políticas urbanas ha puesto de manifiesto que han sido muy numerosos los diferentes instrumentos de gobernanza aplicados para diseñar e implementar políticas urbanas (Martí-Costa \& Tomàs, 2016) y, entre dichos instrumentos, se encuentra la Planificación Estratégica Urbana, que se caracteriza por ser un tipo de planificación de ciudades sustentado en una visión integral y compleja de los diferentes elementos que son necesarios para el desarrollo social y económico de una ciudad y que se soporta en la participación y colaboración de 
los actores públicos y privados. De esta manera, la Planificación Estratégica Urbana es considerada como la aplicación del modelo de gobernanza urbana a la planificación de ciudades (Frewer, 2007; Mäntysalo, Jarenko, Nilsson \& Saglie, 2015) con la finalidad de que los planes de ciudad, como elemento propio de las políticas urbanas de gobernanza, se adapten a las nuevas exigencias que caracterizan a los retos urbanos y sus soluciones, que pasan por que dichos planes sean integrados y estén sustentados, en su diseño e implementación, en el establecimiento de redes de colaboración entre los actores públicos y privados de la ciudad (Desouza \& Flanery, 2013; Harrison \& Hoyler, 2014).

Para la gobernanza urbana y para la Planificación Estratégica Urbana como instrumento, su elemento definitorio y fundamental son las redes de colaboración entre actores públicos y privados para el diseño e implementación de actuaciones. En este sentido, el interés analítico clave para el estudio y comprensión de la gobernanza, y de sus instrumentos, está centrado en las redes de interacción entre los actores (Kirst, Meister y Rowley, 1984; Ansell \& Gash, 2008), es decir, el estudio de las relaciones. Cabe destacar que son muchos los aspectos y características de las redes de relación entre actores en el campo de las políticas públicas que son susceptibles de ser analizados, para los cuales se pueden aplicar diferentes enfoques analíticos (Van Waarden, 1992; Rhodes, 1996; Klijn \& Skelcher, 2007). Uno de estos enfoques es el Análisis de Redes Sociales (ARS), que permite estudiar las propiedades estructurales de las redes obtenidas a partir de la aplicación de un análisis cuantitativo mediante un sistema matricial y el álgebra de grafos para caracterizar las relaciones entre actores (Howlett, 2002; Robins, Lewis \& Wang, 2012).

La investigación que aquí se presenta pretende abordar las tres cuestiones fundamentales destacadas con anterioridad: tiene por objetivo analizar las características de la gobernanza entendida como una red de colaboración entre actores para el diseño e implementación de una acción pública; la gobernanza es estudiada a través de uno de sus instrumentos de política pública, en concreto, se analiza un Plan Estratégico Urbano, el Segundo Plan Estratégico de Málaga; y se centra en el estudio de las propiedades estructurales de la red de gobernanza mediante la aplicación del ARS a la red de colaboración entre actores públicos y privados implicados en la implementación de dicho instrumento.

La estructura del trabajo que se presenta comienza con el establecimiento de un marco teórico entorno a los elementos claves del desarrollo de la investigación: gobernanza, planificación estratégica urbana y aplicación del ARS al estudio de la política. A continuación, se presenta una caracterización del Segundo Plan Estratégico de Málaga para contextualizar los elementos fundamentales que constituyen el núcleo de producción de la información necesaria para analizar y se expone, asimismo, la metodología desarrollada para analizar la red de gobernanza que caracteriza la implementación de los proyectos del plan estratégico, estableciendo la operacionalización del concepto de gobernanza y su medición, la utilización de indicadores 
reticulares para su análisis y el estudio de la relación entre dicha gobernanza y el grado de ejecución de los proyectos. El apartado cuarto se destina a presentar los principales resultados a partir de la aplicación de las técnicas de análisis y la obtención de los indicadores utilizados. Por último, el trabajo termina con las conclusiones de los principales elementos identificados que ayudan a caracterizar el sistema de gobernanza estudiado y aquellas que pueden ser extrapolables a otros casos de estudio similares.

\section{Hacia un marco teórico: gobernanza, planificación estratégica urbana y análisis de redes sociales}

La gobernanza es considerada como una forma particular de gobierno que se encuentra en los sistemas políticos modernos (Börzel, 2002), que tiene que ver mucho más con la solución colectiva de problemas y mucho menos con el dominio (Mayntz, 2002). Gobernanza significa una forma de gobernar más cooperativa, donde las instituciones estatales y no estatales, los actores públicos y privados, participan y a menudo cooperan en la formulación y la aplicación de políticas públicas (Mayntz, 2001). Por tanto, tiene que ver con un estilo particular de gobierno donde el proceso de toma de decisiones tiene un marcado carácter colectivo, determinado por las pautas de interacción entre los actores que participan en dicho proceso, considerando que los actores no son sólo los actores públicos, sino que los actores privados tienen una destacada presencia en todo el proceso. Esta nueva forma de ejercer el gobierno viene caracterizada por la cooperación entre un número importante de actores públicos y privados en la formulación y aplicación de las políticas públicas.

La gobernanza representa un cambio en la estructura de la política (Marin \& Mayntz, 1991; O'Toole \& Meier, 2004), lo que supone un nuevo modelo de gobierno que responde al contexto de fragmentación y complejidad (Le Galès, 1998), y que se materializa en el concepto de red, que se define como un conjunto de actores y las relaciones que se producen entre estos para actuar en un área de política pública específica (Marsh \& Rhodes, 1992; Rhodes, 1997). Desde esta perspectiva, los rasgos básicos de la gobernanza son: (1) La existencia de actores de diverso tipo, de la esfera pública y de la privada, que se relacionan en base a la interdependencia de recursos para obtener un objetivo común que tiene que ver con un problema de un área política concreta. La define, por tanto, una estructura multicéntrica; (2) La horizontalidad de las relaciones entre los actores, la aportación común y en grado de igualdad de recursos básicos, que constituyen la base de interacción entre los mismos, que están en igualdad de condiciones en la red, aunque no en igualdad de posiciones. Se distancia, de este modo, de una relación basada en la jerarquía y en el principio de una autoridad central, con fuerte capacidad de imposición, ya que la base de la relación entre actores es la interdependencia; (3) La autocoordinación, en el sentido de que cada red generará una dinámica de funcionamiento propia que tiene que ser movilizada para que se desarrollen las funciones necesarias que permitan conseguir el objetivo común. Cada red de 
actores tendrá que instrumentar su propia estructura, que le permitirá gestionar el desarrollo de las funciones necesarias para la interacción de los actores; (4) La comunicación que se establece entre los distintos actores que están inmersos en la red de relaciones. Todos los actores deben de disponer de la información y de los conocimientos necesarios y adecuados, de manera que la accesibilidad a los mismos y su calidad se convierte en uno de los elementos básicos para poder desarrollar las interacciones en la red; (5) La confianza y la lealtad son dos máximas que están presentes en la estructura de relaciones que caracteriza a la gobernanza. Son acciones entre actores que no persiguen la maximización de los intereses particulares, sino el beneficio común, condicionado por la incapacidad de poder desarrollar por parte de los actores de manera individual las acciones necesarias para resolver un problema. Esto hace que la lógica imperante sea producir resultados comunes y que, por tanto, los actores no tengan que conseguir beneficios a costa de los otros actores, sino que los beneficios de unos están soportados sobre el beneficio común, propio de toda la red; y (6) La existencia un sistema de reglas, formales e informales, que le es propio, estableciéndose como necesario para su funcionamiento, asignando responsabilidades e instrumentos de procedimiento, que articulan las interacciones necesarias entre los diferentes actores para el desarrollo de las funciones que posibiliten la consecución de las metas comunes. Pero a este respecto, es necesario destacar que cada área de política pública tiene sus propias características, provocando que exista una multiplicidad de patrones políticos de interacción entre actores, lo que dificulta pensar en modelos preestablecidos y considerar que las modalidades estarán adecuadas a cada caso.

La noción de gobernanza urbana adquiere sentido en este contexto de transformación de las formas de gobernar y establecer las políticas públicas, que centran la atención en la solución de problemas/retos colectivos de desarrollo de la ciudad (dimensión sustantiva) y en la importancia de la gestión de la colaboración/cooperación de los actores que intercambian sus recursos en red (dimensión procedimental) (Tellería, 2014). Así, para la gobernanza urbana, el desarrollo de instrumentos que permitan movilizar recursos y organizar la fragmentación (a través de mecanismos de coordinación) se vuelve fundamental (Jouve 2005; Le Galès, 2006; Pinson, 2011). De este modo, en el contexto urbano, la acción pública empieza a ser concebida como el conjunto de dispositivos que permiten movilizar actores y recursos de forma estable, coordinando e integrando intervenciones diversas para alcanzar objetivos definidos colectivamente (Pinson, 2011). Como consecuencia de esta situación, las ciudades se convierten en auténticos laboratorios de innovación social y política.

Entre los mecanismos desarrollados en el marco de la gobernanza urbana destaca la planificación estratégica de ciudades, definida como una de las innovaciones más importantes en la gobernanza urbana y territorial surgida en los últimos años (Tanese et al. 2006). La planificación estratégica constituye un proceso de política pública que promueve la cooperación público-privada y la 
participación de la ciudadanía en la formulación e implementación de la estrategia de desarrollo de las ciudades, que es ampliamente difundido en todo el mundo (Martinelli, 2005; Fernández-Güell, 2007; Farinós, 2010; Martín \& Merinero, 2010; Pascual \& Tarragona, 2009; Romero \& Farinós, 2011). De este modo, el plan estratégico como "instrumento de gobernanza" se ubica, en términos analíticos, en el ámbito de las políticas públicas, puesto que constituye una "intervención directa e intencionada sobre los procesos de toma de decisiones relativos a la provisión de bienes colectivos" (Dente, 2007, p. 9). Asimismo, el proceso de planificación estratégica acompaña el policy making a lo largo de todas sus fases (identificación del problema, inclusión en la agenda, formulación de la política, adopción de la política, implementación de la política, evaluación de la política y finalización -cambio o continuación- de la política), ayudando a gestionar de un modo más - menos estructurado las relaciones entre los actores del territorio para definir (y llevar a la práctica), de forma colectiva, una agenda de desarrollo para la ciudad. Desde esta óptica, la "gestión relacional resulta clave, puesto que, como es sabido, los resultados de los procesos de toma de decisiones sobre políticas públicas dependen fundamentalmente de las interacciones que se establecen entre una pluralidad de actores" (Dente, 2011, p. 18). A partir de todos estos elementos es posible mantener que las políticas urbanas están sufriendo un importante proceso de transformación tanto en su contenido como en sus procesos de elaboración e implementación, y la planificación estratégica de ciudades se presenta como uno de los más claros ejemplos de este nuevo tipo de políticas urbanas (Tomàs \& Martí-Costa, 2011).

Teniendo en cuenta lo indicado, se puede comprobar como la planificación urbana en los últimos años del siglo XX y principios del siglo XXI ha experimentado una transformación en sus modelos y metodologías aplicadas, como una respuesta buscada por los gobiernos locales para adaptarse a los actuales contextos sociales y económicos cada vez más complejos, exigentes e interdependientes territorialmente, donde el nuevo escenario de internacionalización y globalización ha provocado que la tradicional planificación basada en la ordenación física y espacial de las ciudades no sea suficiente para el impulso de políticas públicas de desarrollo urbano. Como respuesta a esta nueva situación, a finales de los años ochenta y principios de los noventa comenzó a ponerse en práctica un nuevo tipo de planificación para las ciudades denominado Planificación Estratégica Urbana (Kemp, 1993; Fernández-Güell, 2007), que, como tal, constituye la aplicación del modelo de gobernanza local a las políticas públicas para impulsar el desarrollo socioeconómico en las ciudades. Los principios básicos de esta nueva modalidad de planificación son los propios del modelo de gobernanza para la elaboración e implementación de políticas públicas, es decir, la participación ciudadana y la cooperación público-privada entre actores de la ciudad como método de adaptación a las nuevas exigencias y demandas que el actual contexto de complejidad impone a la mejora de la calidad de vida en las ciudades (Borja \& Castells, 1997; Pascual, 1999; Elizalde, 2003; Fernández-Güell, 2006; Barton, 2006; Krels, 2007). Así, comenzaron a extenderse en 
Estados Unidos procesos de Planificación Estratégica Urbana en ciudades como San Francisco y Chicago y, posteriormente, en otras ciudades como Ontario, Sydney, Manchester, Londres, Barcelona, Valencia, Ámsterdam, Lisboa, Milán, Turín, Sao Paulo, Rosario, Medellín, etc. La aplicación de procesos de Planificación Estratégica Urbana en estas ciudades ha provocado que gobiernos locales de otras ciudades en distintas regiones del mundo lo hayan empezado a impulsar, surgiendo, asimismo, redes de cooperación entre ciudades con el objetivo de incrementar la colaboración y la producción de conocimiento (CIDEU, RADEUT, URBAL), y el desarrollo de incentivos por distintos niveles de gobierno para impulsar la aplicación de Planes Estratégicos Urbanos en las ciudades.

Tanto en el concepto de gobernanza en general, como en el de gobernanza urbana en particular y como en la planificación estratégica urbana en concreto, la idea central la constituye la red de relaciones que se establecen entre los diferentes actores públicos y privados para definir y llevar a cabo actuaciones de mejora. Por ello, cobra una especial relevancia teórica y analítica lo que se puede denominar como un enfoque centrado en redes. Desde esta perspectiva, se puede mantener que no existe un único enfoque de análisis de las redes de actores en el estudio de la política, sino al contrario, son amplios y variados los enfoques de análisis de redes que se han aplicado al estudio de la política en general y de las políticas públicas en particular (Chaques, 2004). Uno de dichos enfoques de redes es el de la aplicación del ARS al estudio de la política. Dicho enfoque se articula a través de la adopción de principios de la matemática de matrices para obtener características de las redes de relación que permitan abordar el análisis del fenómeno político. Se trata de aplicar el marco teórico-metodológico del ARS al estudio de las interrelaciones para abordar explicaciones de fenómenos sociales complejos, que ha venido siendo aplicado a la investigación en las diferentes Ciencias Sociales (Requena, 2003), y que ha cobrado una especial relevancia analítica en las dos últimas décadas. Son muchos y variados los campos de las diferentes Ciencias Sociales a las que se ha venido aplicando el ARS: sanidad, desarrollo económico, organización empresarial, etc. (Molina, 2001). El objetivo fundamental del ARS está en estudiar las propiedades estructurales de las redes, entendidas estas como un conjunto de nodos (elementos) y los vínculos que se producen entre estos (relaciones) (Wasserman \& Galaskiewicz, 1994; Wasserman \& Faust, 2013). Se trata, por tanto, de poner el centro de interés analítico en las propiedades reticulares de la red, más que en las características individuales de los elementos que harían posibles las relaciones que la sustentan. Así, queda claro que la unidad de análisis es la red y sus propiedades y no las características atributivas de los elementos que la conforman (Marsden y Lin, 1982). No es este el momento ni el lugar para establecer un debate en profundidad sobre las características y los principios teóricos-metodológicos que inspiran este enfoque, ya que existe una importante y dilatada literatura científica sobre el ARS (Burt \& Minor, 1983; Knoke \& Kuklinski, 1982; Scott, 1991; Wellman \& Berkowitz, 1988; Carrington, Scott \& Wasserman, 2005; Jackson, 
2010; Newman, 2010; Scott \& Carrington, 2011) a la que se puede recurrir en cualquier momento para una mayor comprensión del enfoque aquí propuesto. Pero sí es conveniente precisar que desde este enfoque la red se conforma como un conjunto de elementos (personas, grupos, organizaciones, países o acontecimientos) a los que se le denominan nodos y los vínculos específicos que se producen entre ellos a los que denominamos lazos. Se trata de, partiendo de diferentes técnicas de obtención de información, conseguir datos relacionales entre los elementos que conforman la red para aplicar sobre ellos análisis sustentados en álgebra matricial y en operaciones de grafos que nos proporcionarán las propiedades estructurales de la red estudiada, a partir de las medidas de cohesión (centralidad y agrupación) y de equivalencia estructural (Freeman, 2004).

La aplicación del ARS al estudio de la política proviene fundamentalmente de Estados Unidos y consiste en el empleo de complejos y sofisticados métodos de análisis cuantitativos basados en la teoría sociológica del análisis estructural de redes de determinados dominios políticos. Esta línea empírica comenzó en la segunda mitad de los años setenta a través del análisis de los sistemas de influencias de actores para configurar políticas públicas (Laumann \& Pappi, 1976), tuvo su definición más precisa en los años ochenta con el desarrollo de un modelo de análisis sobre ámbitos políticos concretos (Knoke \& Laumann, 1982; Knoke, 1990) y su desarrollo más importante a través de la aplicación del modelo para el análisis de políticas sectoriales como la de salud, energía, laboral y agricultura (Heinz et al., 1993) y alguna aplicación comparativa entre países como fue el caso de la política laboral en Estados Unidos y Alemania (Pappi \& Knoke, 1991). Para la política española cabe destacar el trabajo realizado por el profesor Josep Rodríguez sobre el estudio de la estructura del poder económico en España en el que analiza la estructura de las relaciones entre las principales entidades económicas y políticas españolas (Rodríguez, 2000).

Sin embargo, es importante destacar de cara al interés del trabajo que aquí se presenta, que han sido relativamente escasos los intentos de aplicar el ARS al estudio de la gobernanza, en general, y de la gobernanza urbana, en particular. En los últimos años, la literatura científica sobre la gobernanza se ha centrado especialmente en el análisis de los instrumentos y mecanismos concretos para articular la colaboración y la coordinación entre actores públicos y privados en campos concretos de política pública y, especialmente, en la aplicación de técnicas y sistemas de mecanismos concretos para gestionar las interacciones de cara a mejorar los resultados de esta (Meier \& O’Toole, 2007; Klijn \& Koppenjan, 2012). Sin embargo, a pesar de la escasa aplicación del ARS al estudio de la gobernanza y, especialmente, su aplicación a la gobernanza urbana, sí es importante destacar algunos trabajos aparecidos recientemente en la literatura científica, donde es posible encontrar el análisis de indicadores de centralidad de redes para el estudio y caracterización de la misma (Dente \& Coletti, 2011) y a partir del cual, es posible destacar el interés 
por desarrollar una línea de investigación que aplique el ARS para el estudio y caracterización de la gobernanza.

\section{Elementos metodológicos para el estudio de la gobernanza}

\subsection{La planificación estratégica urbana en Málaga}

Con la inscripción oficial de la Fundación "Centro de Investigaciones Estratégicas y de Desarrollo Económico y Social de Málaga" (CIEDES) el 1 de diciembre de 1995, se sostiene y da continuidad al proceso de planificación estratégica urbana que se había iniciado en 1992 en Málaga, a su elaboración, al seguimiento de su ejecución y a sus sucesivas reelaboraciones y revisiones. Actualmente, son patronos de la Fundación CIEDES el Ayuntamiento de Málaga, la Diputación Provincial, la Junta de Andalucía, la Subdelegación del Gobierno, la Autoridad Portuaria, el Parque Tecnológico de Andalucía (PTA), la Cámara de Comercio, la Confederación de Empresarios de Málaga (CEM), la Unión General de Trabajadores (UGT), Comisiones Obreras (CCOO), la Universidad de Málaga (UMA), la Federación de Asociaciones de Vecinos y Unicaja.

El trabajo realizado por la Fundación CIEDES ha dado como resultado la elaboración, aprobación y posterior seguimiento de dos procesos de planificación estrategia. El Primer Plan Estratégico de Málaga se aprueba en 1996, mientras que el Segundo Plan Estratégico de Málaga se inicia en 2002 y se aprueba en 2006 (Fundación CIEDES, 2006), siendo reformulado en el año 2010 al no poder abordar con garantías las actuaciones propuestas a causa de las crisis, por lo que se disminuye el número de proyectos y de actuaciones a implementar (Fundación CIEDES, 2010).

Con el Segundo Plan Estratégico de Málaga se amplía el ámbito territorial de actuación de la planificación estratégica urbana, pues se pasa de un plan de ciudad a un plan de espacio metropolitano. En un principio, el espacio metropolitano lo constituía, además del municipio de Málaga, los municipios de Torremolinos, Benalmádena, Alhaurín de la Torre, Cártama, Totalán y Rincón de la Victoria, y, posteriormente, se incorporan Alhaurín el Grande, Almogía, Casabermeja, Coín, Colmenar, Fuengirola, Mijas y Pizarra. Así, el espacio metropolitano tiene una extensión de 1390 km² y una población de cerca de 1034000 habitantes, de los cuales algo más de 569000 (55\% del total) son residentes en la ciudad de Málaga, capital de la provincia de su mismo nombre, siendo la segunda capital de provincia más poblada de la Comunidad Autónoma de Andalucía, tras Sevilla, y la sexta de España.

Los dos planes estratégicos se diseñaron bajo la premisa de la coordinación y cooperación administrativa y el consenso ciudadano e interinstitucional en la toma de decisiones, de manera que los proyectos se construyeran con base en una nueva gobernanza del territorio. Cada institución, organismo y/o agente económico y social debía de llevar a cabo sus propios proyectos o ejecutar sus competencias con una misma orientación (Fundación CIEDES, 2015), con el objetivo de 
trabajar en una única dirección de crecimiento. Concretamente, el Segundo Plan Estratégico de Málaga, aprobado en 2006, establece cuatro líneas estratégicas (litoral, cultura, conocimiento y renovación) que se desarrollan a través de diez proyectos estrella, los cuales marcan las grandes claves de trabajo y los objetivos a perseguir, permitiendo incorporar actuaciones llevadas a cabo por cualquier organismo, siempre y cuando, estén orientadas hacia al fin que se persigue con el plan estratégico. A continuación, se describen, brevemente, las cuatro líneas estratégicas y los diez proyectos estrella, puesto que son la base sobre la que se realiza el análisis de las relaciones de gobernanza.

\section{Tabla 1. Líneas estratégicas y proyectos estrellas del II Plan Estratégico de Málaga}

\begin{tabular}{|l|}
\hline 1. Málaga, ciudad litoral \\
1. Mar y metrópoli. Puerto y fachada litoral integrada \\
\hline 2. Málaga, ciudad de la cultura \\
2. Ágora mediterráneo. La ciudad como espacio de la cultura \\
3. Málaga Tur. Una ciudad para el turismo \\
4. Málaga, una ciudad que se enriquece con la diversidad cultural \\
3. Málaga, ciudad del conocimiento \\
5. Málaga Innova. Una ciudad para el conocimiento y la innovación \\
4. Málaga, ciudad renovada \\
6. Metrópoli aeroportuaria. Málaga vértice del transporte internacional \\
7. Integración urbana del Guadalmedina. El nuevo espacio que une a los malagueños \\
8. Málaga y la movilidad metropolitana \\
9. Málaga, ciudad solidaria \\
10. Málaga y la gobernanza local
\end{tabular}

Fuente: elaboración propia a partir del II PEM (Fundación CIEDES, 2006)

El proyecto estrella "Mar y metrópoli. Puerto y fachada litoral integrada" es la concreción de la línea estratégica "Málaga, ciudad litoral". Este proyecto propone, a groso modo, establecer un plan de ordenación de todo el litoral y potenciar el Puerto de Málaga a través de una mejora de la conexión con el Centro Histórico de la ciudad y el fomento de actividades ligadas al mar, la cultura, el turismo y el ocio.

La cultura es entendida como uno de los motores fundamentales de desarrollo en términos sociales, económicos y lúdicos en el plan estratégico. Esta concepción se significa en la línea estratégica "Málaga, ciudad de la cultura", que a su vez se concreta en tres proyectos estrellas. El primer proyecto es "Ágora mediterráneo. La ciudad como espacio de cultura", que propone que el espacio urbano se convierta en un museo abierto en donde se exalte la cultura, poniendo de relieve los modos de vida, costumbres, manifestaciones artísticas, conocimientos y tradiciones malagueñas. Así, pretende convertir el Centro Histórico de la ciudad en un gran museo que exprese dicha apuesta cultural con proyectos integrales de musealización y de arte público. El segundo proyecto 
estrella es "Málaga Tur. Una ciudad para el turismo", que tiene como objetivo consolidar a Málaga como la capital de turismo cultural urbano, haciendo valer su localización geográfica en la Costa del Sol. Este proyecto pretende, igualmente, aprovechar y rentabilizar los trabajos desarrollados años atrás en la mejora de las infraestructuras turísticas y culturales. El último proyecto estrella de esta línea estratégica es "Málaga, una ciudad que se enriquece con la diversidad cultural", que promueve una ciudad inclusiva, en donde la integración social de los inmigrantes se considera un factor de enriquecimiento de la sociedad en todos los niveles.

El proyecto estrella "Málaga Innova. Una ciudad para el conocimiento y la innovación" es la formalización de la línea estratégica "Málaga, ciudad del conocimiento". Este proyecto pretende convertir a la metrópoli malagueña en un espacio con elevada densidad de profesionales relacionados con la investigación, el conocimiento, la cultura y las tecnologías de la información y la comunicación. Para ello, el proyecto recomienda y propone a los planes de ordenación urbana identificar zonas concretas en donde desarrollar los "barrios del conocimiento", que son espacios caracterizados por la complejidad y la diversidad de usos (industriales, comerciales, residenciales, etc.), con incorporación de nuevas tecnologías y centros para facilitar el tejido asociativo, el acceso a recursos, con salas de reuniones, red informática, salas de formación, reprografía, etc.

La línea estratégica "Málaga, ciudad renovada" se considera transversal, incorpora aquellos aspectos no tratados específicamente en las líneas anteriores y se concreta en cinco proyectos estrella. El primer proyecto de esta línea estratégica es "Metrópoli aeroportuaria. Málaga vértice del transporte internacional", que pretende posicionar al aeropuerto de Málaga como un nudo de conexión de repercusión internacional y para ello, se considera imprescindible dotarlo de las infraestructuras, equipamientos e inversiones necesarias (nueva terminal, aparcamientos, segunda pista, etc.). Asimismo, se plantea la necesidad de integrarlo con la red de transporte metropolitano (autobuses, metro, ferrocarril, AVE, autovías, etc.) para establecer una clara intermodalidad entre los mismos. El segundo proyecto estrella es el denominado "Integración urbana del Guadalmedina. El nuevo espacio que une a los malagueños", que se proyecta como "una de las mayores transformaciones urbanas de la historia de la ciudad" (Fundación CIEDES, 2006, p. 201) al actuar sobre buena parte de la estructura urbana, favoreciendo la articulación interna y la accesibilidad del conjunto del territorio. Este proyecto que tiene vocación de convertirse en elemento de centralidad metropolitana al trascender los límites municipales de la ciudad. El tercer proyecto estrella es "Málaga y la movilidad metropolitana", que se plantea con la idea de modular y organizar el movimiento de las personas de manera coordinada y buscando la comodidad, accesibilidad y agilidad en los traslados y transportes del espacio metropolitano. Así, el proyecto propone la realización de un análisis del modelo de ocupación del territorio del área metropolitana y recomienda a los municipios que planifiquen y coordinen los usos de suelo para que la incorporación y distribución de las infraestructuras y los servicios de transportes estén acordes a las 
nuevas necesidades. El cuarto proyecto estrella de esta línea estratégica es "Málaga, ciudad solidaria", que busca, en general, incrementar la calidad de vida y el bienestar de los ciudadanos a través de la equidad social. Se propone, por ejemplo, un plan de acción para la construcción de viviendas de protección oficial que garantice el acceso de los colectivos más necesitados (personas que viven solas, familias monoparentales, jóvenes, discapacitados, etc.) o el fomento de la idea de "la ciudad es de todos" (Fundación CIEDES, 2006, p. 211) por medio de la eliminación de barreras físicas e ideológicas. El quinto y último proyecto estrella de esta línea estratégica es "Málaga y la Gobernanza Local", que, apuesta por una necesaria modernización de las administraciones para permitir la participación de los ciudadanos y demás organismos en la gestión de la ciudad, buscando que la gestión coordinada y consensuada entre los actores del territorio sea la base de la toma de decisiones.

\subsection{Definiendo la gobernanza en el Segundo Plan Estratégico de Málaga}

Es necesario tener presente que el número de coordinaciones (interacciones) entre los actores para llevar a cabo una acción pública es un elemento fundamental que facilita el correcto funcionamiento de los procesos de gobernanza en la implementación de políticas públicas (Falleti \& Lynch, 2009; Busetti \& Dente, 2018). Sobre la base de este elemento se plantea el sistema de gobernanza impulsado en el marco del Segundo Plan Estratégico de Málaga.

En el año 2014, la Fundación CIEDES decide evaluar si desde el 2006 el camino recorrido realmente había sido el más adecuado y si se habían obtenido los frutos deseados con la planificación estratégica. Se detecta, durante este proceso, que no habían desarrollado vías de concertación o de información sobre la necesidad de acuerdos y cómo llevarlos a cabo entre las instituciones. Por ello, desarrollaron un instrumento que facilitaba la colaboración entre los patronos, que denominaron "Matriz de relación de agentes" (Fundación CIEDES, 2015). Esta es una matriz de doble entrada, donde se sitúan los patronos en ambos ejes y el contenido de la misma se basa en la información que se recoge de cada patrono sobre las actuaciones que estarían interesados en coordinar con otros patronos. De esta forma, en la horizontal de la matriz se presentaban las propuestas de coordinación de actuaciones que cada patrono le hacía a cada uno de los demás, mientras que, en la vertical, cada patrono, podía ver las propuestas que los demás le hacían.

Las propuestas de coordinación de las actuación debían de cumplir con unos criterios previamente establecidos: (1) Todos los proyectos debían tener una visión con alguna de las líneas del plan estratégico y una visión o marco superior que marcase objetivos/estrategias; (2) Para proyectos materiales o con ejecución física se estipulaba que el importe fuera superior a 250000 euros y que fueran de disfrute colectivo y/o abierto a otras instituciones y personas; y (3) Para proyectos inmateriales o sin ejecución física, los proyectos incluidos debían tener una duración superior a un año y estar coordinados con al menos otro organismo (Fundación CIEDES, 2015). 
En la Tabla 2 se recoge el número de veces en los que los patronos se coordinaron para la implementación de actuaciones durante el periodo 2007-2013. El contenido de la misma está basado en la información que se recoge de cada patrono sobre las acciones y proyectos que se pusieron en marcha. Desde el punto de vista metodológico, se obtiene una matriz de adyacencia, es decir, una matriz cuadrada con la misma cantidad de filas que de columnas, que es una cuestión base analítica fundamental para poder aplicar el ARS. Asimismo, es una matriz ponderada o valorada, puesto que se completa con distintas cifras en función de la mayor o menor cantidad de actuaciones coordinadas entre los patronos. Igualmente, desde un punto de vista relacional, es una matriz simétrica, puesto que la relación entre los patronos se da manera bidireccional.

Tabla 2. Matriz de resultados de la gobernanza (ponderada)

\begin{tabular}{|c|c|c|c|c|c|c|c|c|c|c|c|c|c|}
\hline & Ayto. & Puerto & Cámara & CEM & $\mathrm{CCOO}$ & Junta & Diputación & Vecinos & Subdeleg. & PTA & Unicaja & UMA & UGT \\
\hline Ayło. & & 4 & 14 & 15 & 7 & 38 & 9 & 6 & 13 & 7 & 13 & 16 & 6 \\
\hline Puerto & 4 & & 4 & 2 & 2 & 6 & 2 & 2 & 4 & 2 & 2 & 2 & 2 \\
\hline Cámara & 14 & 3 & & 13 & 5 & 7 & 6 & 3 & 4 & 4 & 6 & 7 & 5 \\
\hline CEM & 15 & 2 & 13 & & 7 & 9 & 8 & 3 & 4 & 3 & 10 & 11 & 7 \\
\hline $\mathrm{CCOO}$ & 7 & 2 & 5 & 7 & & 8 & 3 & 4 & 7 & 2 & 4 & 3 & 11 \\
\hline Junta & 38 & 6 & 7 & 9 & 8 & & 7 & 2 & 19 & 10 & 9 & 20 & 5 \\
\hline Diputación & 9 & 2 & 6 & 8 & 3 & 7 & & 2 & 2 & 2 & 6 & 6 & 3 \\
\hline Vecinos & 6 & 2 & 3 & 3 & 4 & 2 & 2 & & 2 & 3 & 3 & 3 & 4 \\
\hline Subdeleg. & 13 & 4 & 4 & 4 & 7 & 19 & 2 & 2 & & 3 & 4 & 9 & 5 \\
\hline PTA & 7 & 2 & 4 & 3 & 2 & 10 & 2 & 3 & 3 & & 4 & 8 & 3 \\
\hline Unicaja & 13 & 2 & 6 & 10 & 4 & 9 & 6 & 3 & 4 & 4 & & 10 & 4 \\
\hline UMA & 16 & 2 & 7 & 11 & 3 & 20 & 6 & 3 & 9 & 8 & 10 & & 6 \\
\hline UGT & 6 & 2 & 5 & 7 & 11 & 5 & 3 & 4 & 5 & 3 & 4 & 6 & \\
\hline TOTAL & 148 & 33 & 78 & 92 & 63 & 140 & 56 & 37 & 76 & 51 & 75 & 101 & 61 \\
\hline
\end{tabular}

Fuente: adaptado de Fundación CIEDES (2015)

Asimismo, en la Tabla 2 se puede observar el total de actuaciones coordinadas por los patronos. El Ayuntamiento de Málaga, con un total de 148 es el patrono más que se coordinó, seguido de la Junta de Andalucía y la UMA que lo hicieron en 140 y 101 ocasiones respectivamente. La CEM con 92 coordinaciones, la Cámara de Comercio con 78, la Subdelegación del Gobierno con 76 y Unicaja con 75 son los patronos siguientes. Los sindicatos CCOO y UGT se coordinaron en 63 y 61 respectivamente. Los patronos con menos coordinaciones fueron la Diputación Provincial con 56, el PTA con 51, la Federación de Asociaciones de Vecinos con 37 y la Autoridad Portuaria con 33.

\subsection{Indicadores reticulares (ARS) para el análisis de la Gobernanza}

En la siguiente tabla aparecen los indicadores reticulares que se han utilizado para analizar las características de la gobernanza del Segundo Plan Estratégico de Málaga aplicando el ARS. Hay que tener presente que el análisis que se hace de estos indicadores se plantea en términos de redes sociocéntricas o completas (Molina, 2001), es decir, un análisis de las relaciones que se 
establecen entre todos los actores que intervienen en la implementación del Segundo Plan

Estratégico de Málaga.

\section{Tabla 3. Descripción de los Indicadores utilizados}

\begin{tabular}{|c|c|}
\hline Estructura reticular & $\begin{array}{l}\text { Es la representación gráfica de las relaciones que se producen entre los diferentes } \\
\text { actores mediante el establecimiento de vínculos (enlaces) entre actores (nodos) } \\
\text { implicados. Esta representación gráfica permite ver la estructura de la intensidad de las } \\
\text { relaciones entre los actores. }\end{array}$ \\
\hline $\begin{array}{l}\text { Densidad } \\
\text { (Density) }\end{array}$ & $\begin{array}{l}\text { Calcula el número de relaciones existentes en relación al número total de posibles } \\
\text { relaciones. Para valores ponderados, la densidad calcula la suma total de los valores de } \\
\text { la matriz, dividido por la cantidad máxima de relaciones posibles (sin ponderación). Es } \\
\text { decir, en este caso se mide la ponderación media de la matriz (considerando también el } \\
\text { cero como una ponderación posible). Indica cómo es la conectividad de la red. }\end{array}$ \\
\hline $\begin{array}{l}\text { Grad } \\
\text { (Degr }\end{array}$ & $\begin{array}{l}\text { En el caso de matrices simétricas, es decir, con relaciones bidireccionales, calcula el } \\
\text { número de actores a los cuales un actor está directamente unido. Adicionalmente, su } \\
\text { "Grado de centralidad normalizado", es la centralidad observada del actor dividida por } \\
\text { la cantidad máxima de relaciones que podría haber desarrollado y multiplicada por } 100 \\
\text { para llevarlo a porcentaje (es un porcentaje individual por lo que la suma de todos los } \\
\text { actores no tiene que ser } 100 \% \text { ). } \\
\text { En el caso de matrices no simétricas, el Grado de Centralidad tiene dos componentes, el } \\
\text { "Grado de Centralidad de Entrada" (in-degree) y el "Grado de Centralidad de Salida" } \\
\text { (out-degree). El grado de centralidad de entrada es el número de lazos relacionales } \\
\text { recibidos por un actor, mientras que el de salida el número de lazos relacionales } \\
\text { emitidos. Adicionalmente, para ambos componentes, su "Grado de centralidad } \\
\text { normalizado", es la centralidad observada del actor dividida por la cantidad máxima de } \\
\text { relaciones que podría haber recibido o emitido y multiplicada por } 100 \text { para llevarlo a } \\
\text { porcentaje. }\end{array}$ \\
\hline $\begin{array}{l}\text { Grado } \\
\text { (Centra }\end{array}$ & $\begin{array}{l}\text { Expresa el grado de similitud de una red con otra tipo estrella del mismo tamaño (la red } \\
\text { estrella es la más centralizada). El indicador aporta un dato como proporción que debe } \\
\text { ser multiplicado por } 100 \text { para convertirlo en porcentaje. Así, cuanto más se acerque el } \\
\text { porcentaje a } 100, \text { la red será más centralizada y cuanto más se aleje, más pluricéntrica. }\end{array}$ \\
\hline $\begin{array}{l}\text { Grad } \\
\text { (Betw }\end{array}$ & $\begin{array}{l}\text { Para un actor " } \mathrm{A} \text { " es el número de caminos que pasan por el mismo para unir a otros } \\
\text { dos actores "B" y " } \mathrm{C} \text { ", dividido por el total de caminos posibles que estos últimos } \\
\text { actores tienen de conexión a través de un tercero (siempre teniendo en cuenta el camino } \\
\text { más corto, es decir, la distancia geodésica). La medida de intermediación de un actor se } \\
\text { obtiene al contar las veces que éste aparece en los caminos geodésicos que conectan a } \\
\text { todos los pares de actores en la red (a estos actores se les denomina "actores puente"). } \\
\text { Adicionalmente, el "Grado de Intermediación Normalizado" de un actor es su grado de } \\
\text { intermediación dividido por el máximo grado de intermediación que podría obtener en } \\
\text { esta red, expresado en porcentaje. }\end{array}$ \\
\hline $\begin{array}{l}\text { Grado } \\
\text { (Close }\end{array}$ & $\begin{array}{l}\text { Es la capacidad de un actor de llegar a todos los actores de la red. Se calcula al dividir } \\
\text { el valor de "uno" entre la suma de los geodésicos (las distancias más cortas que un } \\
\text { actor debe recorrer para llegar a los demás actores) y multiplicarlo por "mil". Valores } \\
\text { altos de cercanía indican una mejor capacidad de los actores para conectarse con los } \\
\text { demás actores de la red. El Grado de Cercanía sólo es posible aplicarlo en matrices } \\
\text { simétricas. }\end{array}$ \\
\hline $\begin{array}{l}\text { Poder de Bonacich } \\
\text { (Power Bonacich) }\end{array}$ & $\begin{array}{l}\text { Calcula las relaciones de cada actor tiene con sus contactos directos, más las relaciones } \\
\text { de estos con sus contactos directos y así, sucesivamente, hasta completar la red. } \\
\text { Diferencia entre centralidad y poder. Así, un actor " } A \text { " que está bien conectado a otros } \\
\text { actores, que a su vez están bien conectados, hacen más central a " } \mathrm{A} \text { ", pero no } \\
\text { poderoso. Por el contrario, si el actor " } \mathrm{A} \text { " está conectado a otros actores que no están } \\
\text { bien conectados, hace que el primero sea más poderoso, porque estos últimos actores } \\
\text { dependen del mismo para poder conectarse a la red. Determina que la centralidad de } \\
\text { un actor está en función de la cantidad de conexiones directas que éste tiene y cuántas } \\
\text { conexiones tienen los actores a los que está conectado. } \\
\text { La fórmula es la misma para calcular la centralidad que para calcular el poder, lo que } \\
\text { cambia es el valor de beta ( } \beta \text { ), que es el peso que se le da a las relaciones de ego: (1) } \\
\text { Para calcular la centralidad de los actores se usa un valor positivo de beta ( } \beta \text { ), lo que } \\
\text { quiere decir que se le da un peso positivo a las relaciones directas; y (2) Para calcular el } \\
\text { poder de los actores se usa un valor negativo de beta }(\beta) \text {, lo que quiere decir que se le } \\
\text { da un peso negativo a las relaciones directas. }\end{array}$ \\
\hline
\end{tabular}

Fuente: elaboración propia 
La representación gráfica de la estructura reticular de la intensidad relacional entre los patronos del Segundo Plan Estratégico de Málaga ha sido desarrollada mediante el software VISONE. Los indicadores, tanto la densidad como los diferentes indicadores de centralidad, se han calculado con el software UCINET 6 e igualmente, se ha utilizado el software VISONE para representarlos gráficamente.

\subsection{Construcción de la red de dos modos}

Una red de dos modos, también denominada red de afiliación, es una estructura relacional en donde un determinado universo se refiere a otro universo distinto, que para el caso analizado, corresponde a los patronos (primer universo) y a las líneas estratégicas en las cuales los primeros se coordinan (segundo universo). Se ha recurrido aquí a la red de dos modos para comprobar si existe una correspondencia entre la gobernanza y el grado de ejecución de las líneas. Los datos de las coordinaciones entre patronos por cada línea estratégica se han obtenido contabilizado todas las actuaciones en donde al menos dos patronos se han coordinado en el diseño o en la implementación de los proyectos estrella y, consecuentemente, de las líneas estratégicas. El resultado total de las coordinaciones entre patronos se muestra en la "Matriz de intensidad de coordinaciones entre patronos por línea estratégica" de la Tabla 11. Así, con los datos de la matriz se obtiene la red de dos modos de la Figura 5. Este grafo permite una doble lectura, ya que, por un lado, posibilita ver en qué líneas estratégicas se producen las mayores coordinaciones y por otro, visibiliza cuáles son los patronos que más se coordinan y en qué líneas estratégicas lo hacen.

El desarrollo pormenorizado de la matriz y de la red de dos modos será desarrollado en el apartado 5 .

\section{Resultados del análisis ARS de la gobernanza del segundo plan estratégico de Málaga}

En este apartado se presentan los resultados obtenidos de la aplicación de los diferentes indicadores reticulares utilizados para estudiar la gobernanza, que se ha estructurado en dos partes: primero, se exponen los indicadores realizados con datos ponderados y luego, se presentan los indicadores realizados con datos dicotomizados. Para finalizar el apartado, se muestra la aplicación de la técnica de la matriz de dos modos para analizar la relación entre la gobernanza y el grado de ejecución de las líneas estratégicas.

\subsection{Indicadores con datos ponderados}

a) Estructura reticular de la intensidad relacional de la Gobernanza

Para realizar el análisis de la estructura reticular de la gobernanza se ha seguido el mismo criterio que el utilizado por la propia Fundación CIEDES en el documento denominado "Evaluación del II Plan Estratégico de Málaga 2007-2014 y propuesta marco para una Estrategia Integrada de 
Desarrollo Sostenible Málaga 2020" del año 2015. Así, considerando los resultados obtenidos en la "Matriz de resultados de la gobernanza (ponderada)", se establecen unos rangos de valores, con el objetivo de clasificar las coordinaciones en intensas (20 o más), fluidas (entre 10 y 19), moderadas (entre 6 y 9), débiles (entre 3 y 5) y esporádicas (2 o menos), en función de las veces en que los patronos se han coordinado para implementar una actuación. El resultado de dicho agrupamiento da un valor de intensidad de la relación que se representa en la Tabla 4.

Tabla 4. Matriz de intensidad de las relaciones entre los patronos

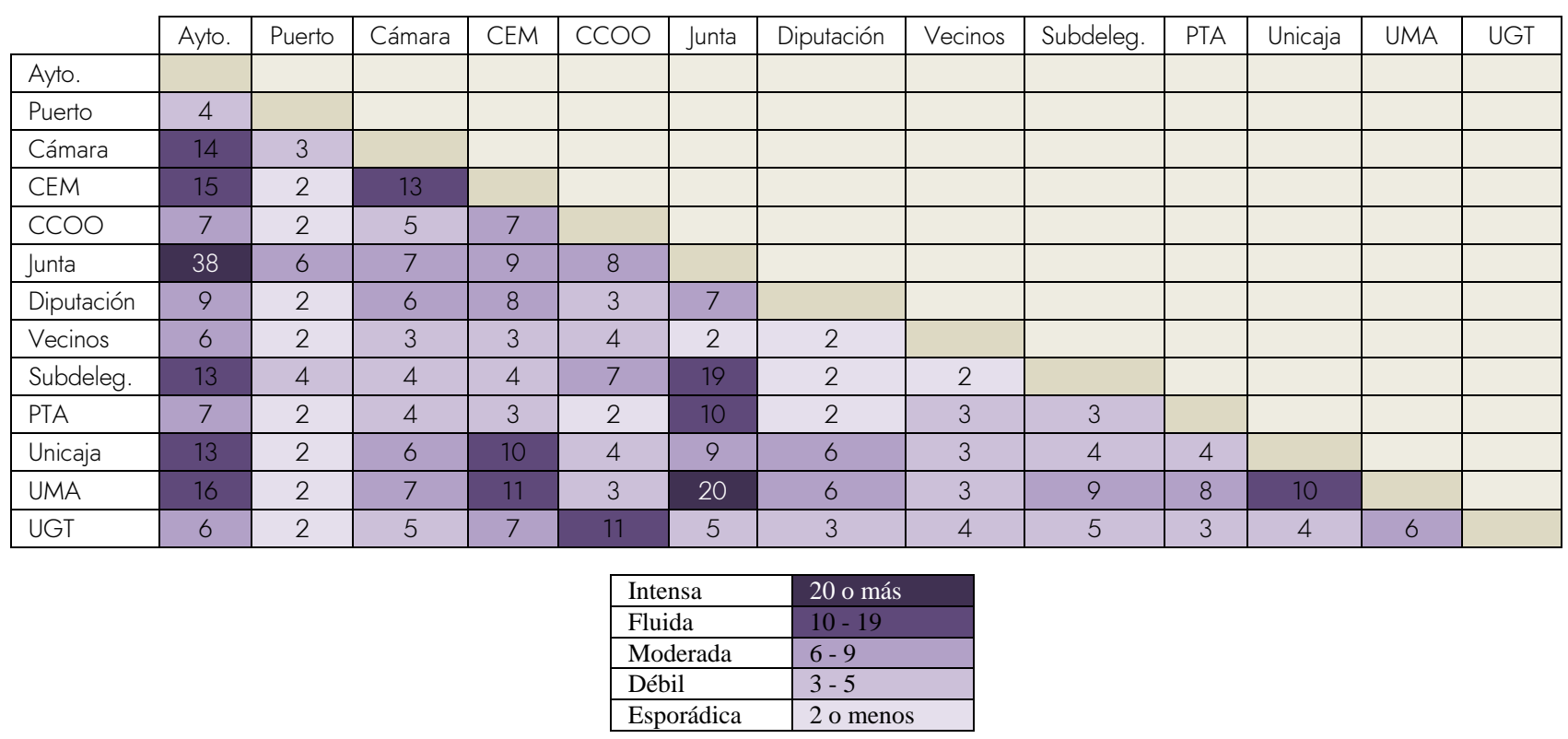

Fuente: adaptado de Fundación CIEDES (2015)

Esta intensidad relacional tiene su reflejo visual en el grafo denominado "Red de intensidad de las relaciones entre los patronos" de la Figura 1. Para la elaboración de este, se han tenido en cuenta solamente las relaciones moderadas, fluidas e intensas, al entender que las relaciones débiles y esporádicas, es decir, con cinco coordinaciones o menos, tienen poco impacto en términos de incidencia sobre las actuaciones. Esto significa que, desde el punto de vista del análisis realizado, para que exista relación de gobernanza tiene que haber como mínimo seis coordinaciones entre los patronos en la implementación de una actuación o proyecto.

La estructura de la red de intensidad permite visualizar que las relaciones intensas surgen entre patronos públicos, esto es, la Junta de Andalucía con el Ayuntamiento de Málaga y con la UMA. Hay un total de 12 relaciones fluidas, destacando, con la mitad de estas, las acontecidas entre patronos públicos con privados, mientras que las relaciones entre privado-privado y público-público son tres en ambos casos. Por último, las relaciones moderadas son un total de 23 y se distribuyen de la siguiente manera: cinco público-público, tres privado-privado y quince público-privado. 
Figura 1. Red de intensidad de las relaciones entre los patronos

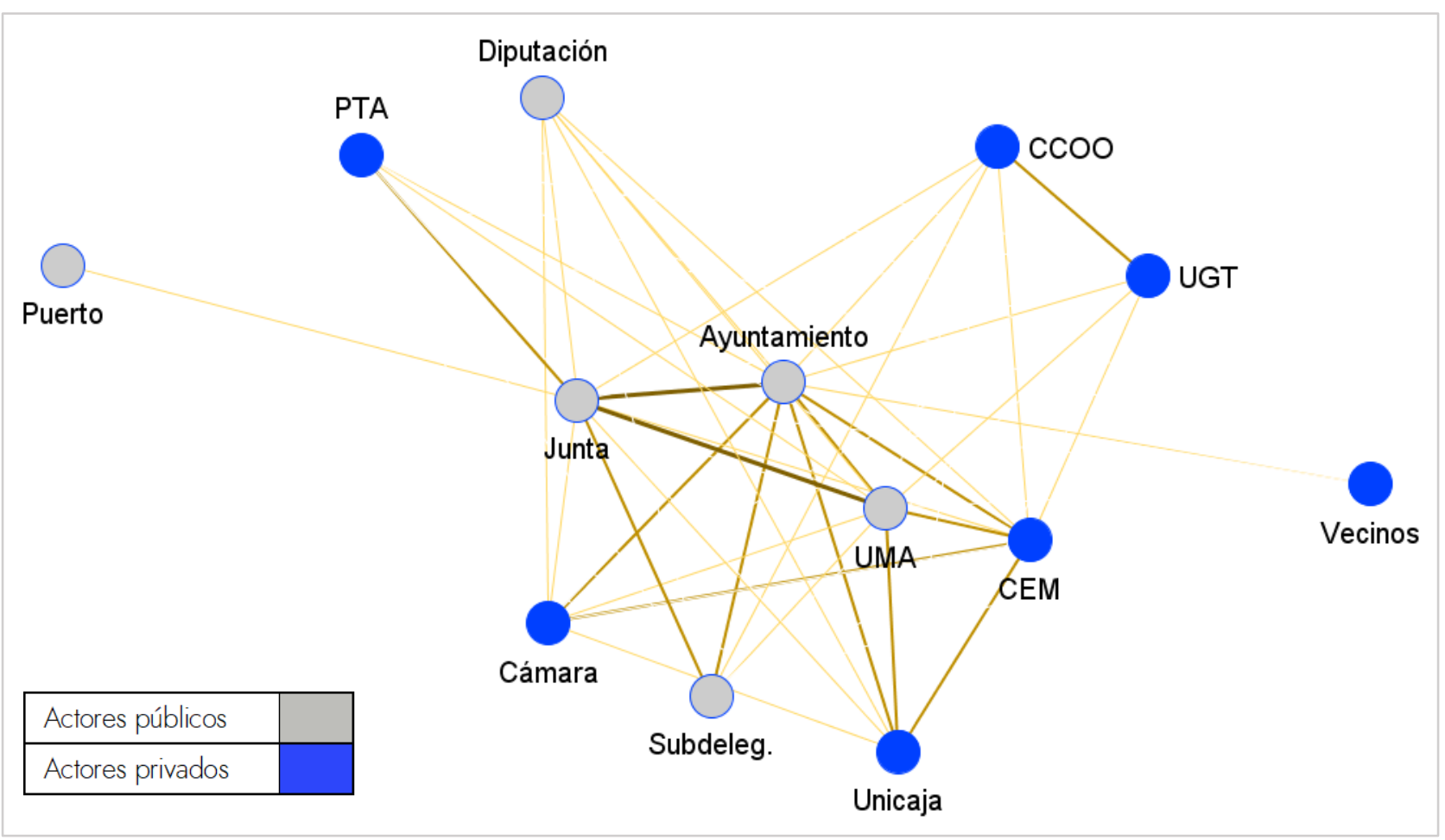

Fuente: elaboración propia con VISONE

Esta red de intensidad permite una primera interpretación del posicionamiento de los patronos en la estructura reticular. Se puede observar que los que tienen un mayor número de relaciones se localizan en zonas centrales de la red, mientras que a medida que van teniendo menos, se van situando hacia los extremos. El centro de la red está dominado por patronos de carácter público, siendo estos el Ayuntamiento de Málaga, la Junta de Andalucía y la UMA, que coindicen, además, con los patronos que tienen las relaciones intensas de coordinación. La CEM, como patrono privado, también tiene una posición relativamente céntrica en la estructura reticular. Es destacable que, entre los cuatro patronos mencionados y estos, con la Subdelegación del Gobierno, la Cámara de Comercio y Unicaja, ocurre la totalidad de relaciones fluidas, a excepción de las que se suceden entre la Junta y el PTA, y entre los sindicatos. Por lo tanto, se puede decir que el núcleo de las relaciones intensas y fluidas se da entre estos siete patronos, existiendo un equilibrio entre patronos de carácter público y privado. La Diputación Provincial, aunque está conectada con cinco patronos, se incorporada a la red de manera moderada, al igual que CCOO y UGT. La Federación de Asociaciones de Vecinos y la Autoridad Portuaria sólo se conectan a la red a través de una relación y, además, esta es, en ambos casos, de carácter moderado, lo que hace que aparezcan alejados en la estructura relacional. 


\section{b) Densidad de la red de Gobernanza}

El indicador de cohesión de Densidad de la red está íntimamente relacionado con la intensidad de las relaciones, ya que su cálculo se realiza sobre la suma total de los valores ponderados de la matriz, dividido por la cantidad máxima de relaciones posibles (sin ponderación). Así, la densidad de la red analizada es de un $64.8 \%$, lo que significa que, de cada 100 relaciones posibles entre los patronos, se han producido casi 65. Dada las características de esta red, que requiere de la implicación de múltiples actores que responden a ámbitos competenciales diferentes, que tienen objetivos distintos (y a veces contradictorios) y con estructuras organizativas heterogéneas, se considera que la red tiene una conectividad alta, con relaciones fuertes. Esta alta densidad de la red permite afirmar, en un primer acercamiento, que el desarrollo y la implementación de las actuaciones recogidas en el plan estratégico se han fundamentado en la idea de coordinación y cooperación administrativa e interinstitucional, base para la gobernanza del territorio.

\subsection{Indicadores con datos dicotomizados}

Para el cálculo de los indicadores de centralidad es necesario dicotomizar la matriz de gobernanza ponderada, siendo esto una exigencia matemática, puesto que dichos indicadores no pueden obtenerse sobre datos ponderados. Para la dicotomización se ha operado de la misma manera que para la realización del grafo de la "Red de intensidad de las relaciones entre los patronos", es decir, se ha considerado que para que exista relación de gobernanza tiene que haber como mínimo seis coordinaciones entre los patronos, esto es, se han tenido en cuenta únicamente las relaciones moderadas, fluidas e intensas. De esta manera, a las relaciones consideradas se les asigna un "uno" en la matriz, lo que significa que hay relación, mientras que a las coordinaciones débiles y esporádicas se les asigna un "cero", lo que representa que no hay relación. El resultado de la dicotomización se presenta en la Tabla 5.

Tabla 4. Matriz de resultados de la gobernanza (dicotomizada)

\begin{tabular}{|c|c|c|c|c|c|c|c|c|c|c|c|c|c|}
\hline & Ayto. & Puerto & Cámara & CEM & $\mathrm{CCOO}$ & Junta & Diputación & Vecinos & Subdeleg. & PTA & Unicaja & UMA & UGT \\
\hline Ayto. & 0 & 0 & 1 & 1 & 1 & 1 & 1 & 1 & 1 & 1 & 1 & 1 & 1 \\
\hline Puerto & 0 & 0 & 0 & 0 & 0 & 1 & 0 & 0 & 0 & 0 & 0 & 0 & 0 \\
\hline Cámara & 1 & 0 & 0 & 1 & 0 & 1 & 1 & 0 & 0 & 0 & 1 & 1 & 0 \\
\hline CEM & 1 & 0 & 1 & 0 & 1 & 1 & 1 & 0 & 0 & 0 & 1 & 1 & 1 \\
\hline $\mathrm{CCOO}$ & 1 & 0 & 0 & 1 & 0 & 1 & 0 & 0 & 1 & 0 & 0 & 0 & 1 \\
\hline Junta & 1 & 1 & 1 & 1 & 1 & 0 & 1 & 0 & 1 & 1 & 1 & 1 & 0 \\
\hline Diputación & 1 & 0 & 1 & 1 & 0 & 1 & 0 & 0 & 0 & 0 & 1 & 1 & 0 \\
\hline Vecinos & 1 & 0 & 0 & 0 & 0 & 0 & 0 & 0 & 0 & 0 & 0 & 0 & 0 \\
\hline Subdeleg. & 1 & 0 & 0 & 0 & 1 & 1 & 0 & 0 & 0 & 0 & 0 & 1 & 0 \\
\hline PTA & 1 & 0 & 0 & 0 & 0 & 1 & 0 & 0 & 0 & 0 & 0 & 1 & 0 \\
\hline Unicaja & 1 & 0 & 1 & 1 & 0 & 1 & 1 & 0 & 0 & 0 & 0 & 1 & 0 \\
\hline UMA & 1 & 0 & 1 & 1 & 0 & 1 & 1 & 0 & 1 & 1 & 1 & 0 & 1 \\
\hline UGT & 1 & 0 & 0 & 1 & 1 & 0 & 0 & 0 & 0 & 0 & 0 & 1 & 0 \\
\hline
\end{tabular}

Fuente: elaboración propia a partir de Fundación CIEDES (2015) 
Específicamente, los indicadores de centralidad utilizados han sido el Grado de Centralidad (Degree), el Grado de Centralización de la red (Centralization), el Grado de Intermediación (Betweenness), el Grado de Cercanía (Closeness) y el Poder de Bonacich (Power Bonacich), siendo estos los principales indicadores utilizados para el análisis de redes de políticas públicas.

a) Grado de Centralidad y de Centralización

El indicador del Grado de Centralidad muestra las relaciones directas de cada uno de los patronos. En la Tabla 5 se pueden observar los resultados de dicho cálculo ordenados según su centralidad de mayor o menor. En la primera columna se aprecian las relaciones directas, mientras que en la segunda se muestra el "Grado de centralidad normalizado", es decir, las relaciones directas de cada patrono dividida por la cantidad máxima de relaciones que podría haber desarrollado y multiplicada por 100. Así, se puede apreciar que el Ayuntamiento de Málaga es el patrono con mayor grado de centralidad (0.917), seguido de la Junta de Andalucía (0.833), la UMA (0.750) y la CEM (0.667). En una posición intermedia están la Diputación Provincial, la Cámara de Comercio y la entidad bancaria Unicaja, todos ellos con una centralidad de 0.500 , lo que significa que tienen la mitad de las relaciones directas posibles. El sindicato CCOO, con 0.417 es el siguiente patrono y finalmente, los de menor centralidad son UGT (0.033), la Subdelegación del Gobierno (0.033), el PTA (0.250), la Federación de Asociaciones de Vecinos (0.083) y la Autoridad Portuaria (0.083). El grafo resultante del cálculo de la centralidad se muestra en la "Red de Centralidad" de la Figura 2, que posiciona a los patronos en una circunferencia con círculos concéntricos y así, cuanto más central sea el patrono, más hacia el interior de la misma se situará.

Tabla 5. Grado de Centralidad

\begin{tabular}{|c|l|c|c|}
\hline \multicolumn{2}{|l|}{ Patronos } & Centralidad & Normalizada \\
\hline 1 & Ayuntamiento de Málaga & 11 & 0.917 \\
\hline 2 & Junta de Andalucía & 10 & 0.833 \\
\hline 3 & UMA & 9 & 0.750 \\
\hline 4 & CEM & 8 & 0.667 \\
\hline 5 & Diputación Provincial & 6 & 0.500 \\
\hline- & Cámara de Comercio & 6 & 0.500 \\
\hline- & Unicaja & 6 & 0.500 \\
\hline 8 & CCOO & 5 & 0.417 \\
\hline 9 & UGT & 4 & 0.333 \\
\hline- & Subdelegación del Gobierno & 4 & 0.333 \\
\hline 11 & PTA & 3 & 0.250 \\
\hline 12 & Vecinos & 1 & 0.083 \\
\hline- & Autoridad Portuaria & 1 & 0.083 \\
\hline
\end{tabular}

Fuente: elaboración propia. UCINET 6 
Figura 2. Red de Centralidad

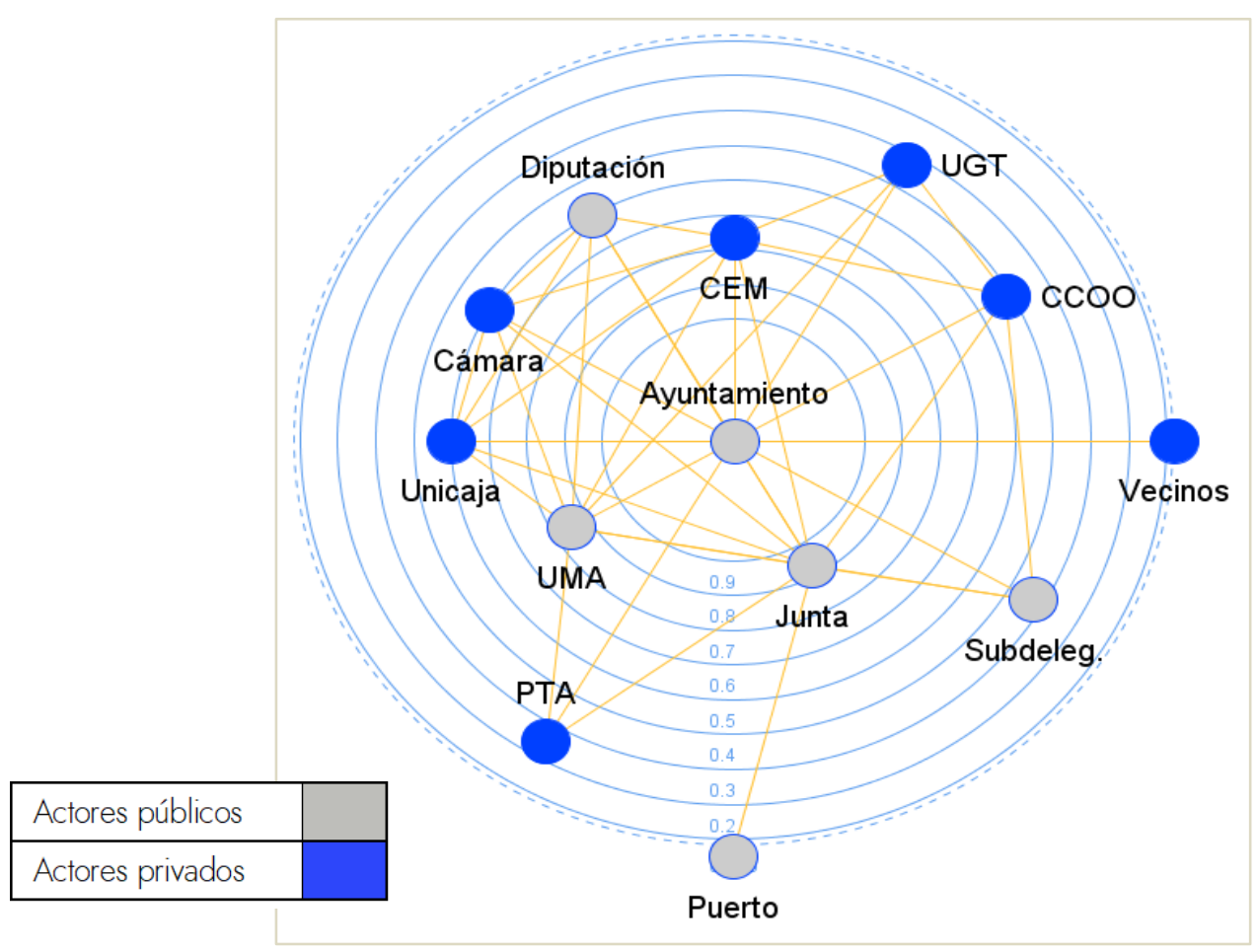

Fuente: elaboración propia con VISONE

El hecho de que existan varios patronos con alta centralidad tiene su reflejo en el Grado de Centralización de la red, que adicionalmente se proporciona cuando se calcula la centralidad, y que para la red analizada muestra una centralización de un 52.2\%. Considerando que un $100 \%$ indica la máxima centralización posible (red tipo estrella), el dato para la red de gobernanza estudiada se asemeja más a una red pluricéntrica, en donde varios actores ejercen como actores centrales. Este hecho está en consonancia con lo pretendido desde la Fundación CIEDES, en cuanto que ha conseguido crear una red en la que son varios los patronos que ejercen un papel central en la misma, distribuyéndose la importancia y el "poder" entre los mismos. Esto se puede observar en la red de centralidad de la Figura 2, en donde el Ayuntamiento de Málaga, la Junta de Andalucía y la UMA comparten lugares centrales, pudiendo ejercer de "líderes" dentro de la configuración estructural de la red. Además, muchos de los patronos se sitúan en una posición relativamente cercana al centro de la red, sólo apareciendo alejados la Subdelegación del Gobierno, el PTA, la Federación de Asociaciones de Vecinos y la Autoridad Portuaria.

b) Grado de Intermediación

El Grado de Intermediación muestra algunas características diferenciales con respecto a la centralidad, lo que permite profundizar en el análisis de la estructura la red de gobernanza analizada. Un dato que destacar es que casi la mitad de los patronos no tienen capacidad de intermediación (Diputación Provincial, Cámara de Comercio, Unicaja, PTA, Federación de 
Asociaciones de Vecinos y la Autoridad Portuaria), por lo que son prescindibles a la hora de conectar a un par de patronos. Otra característica destacable es que los cuatro patronos más centrales según el grado de centralidad (Ayuntamiento de Málaga, Junta de Andalucía, UMA y CEM), son también los más centrales según el grado de intermediación, manteniendo la misma posición jerárquica en la red. Sin embargo, el peso de la corporación local y de la administración autonómica es ahora mayor, puesto que tienen un "Grado de intermediación normalizado" de 27197 y 23662 , mientras la universidad y los empresarios lo tienen de 7955 y 4091 respectivamente. En términos de estructura de la red, esto significa que los dos primeros patronos tienen mucha más capacidad de intermediar entre un par de actores, por lo que se convierten aún en más centrales. Otro dato relevante que muestra este indicador es que la Diputación Provincial, la Cámara de Comercio y Unicaja, que tienen la mitad de las relaciones directas posibles, situándose como los quintos patronos con mayor grado de centralidad, no tienen ninguna capacidad de intermediar, por lo que, igualmente, pierden centralidad. El sindicato CCOO que es el octavo patrono considerando el grado de centralidad, es el quinto es cuanto a intermediación (1.641), mientras que UGT y la Subdelegación del Gobierno que son novenos teniendo en cuenta el primero, son sextos considerando el segundo (0.033).

Tabla 6. Grado de Intermediación

\begin{tabular}{|c|l|c|c|}
\hline \multicolumn{2}{|l|}{ Patronos } & Intermediación & Normalizada \\
\hline 1 & Ayuntamiento de Málaga & 17.950 & 27.197 \\
\hline 2 & Junta de Andalucía & 15.617 & 23.662 \\
\hline 3 & UMA & 5.250 & 7.955 \\
\hline 4 & CEM & 2.700 & 4.091 \\
\hline 5 & CCOO & 1.083 & 1.641 \\
\hline 6 & UGT & 0.200 & 0.303 \\
\hline- & Subdelegación del Gobierno & 0.200 & 0.303 \\
\hline 7 & Diputación Provincial & 0.000 & 0.000 \\
\hline- & Cámara de Comercio & 0.000 & 0.000 \\
\hline- & Unicaja & 0.000 & 0.000 \\
\hline- & PTA & 0.000 & 0.000 \\
\hline- & Vecinos & 0.000 & 0.000 \\
\hline- & Autoridad Portuaria & 0.000 & 0.000 \\
\hline
\end{tabular}

Fuente: elaboración propia a partir con UCINET 6

Los resultados del grado de intermediación ponen de manifiesto que el Ayuntamiento de Málaga y la Junta de Andalucía tienen un papel principal en la estructura de la red, incidiendo directamente en el funcionamiento de la gobernanza del plan estratégico, puesto que la gran mayoría de las relaciones que se dan entre patronos se facilitan gracias a que estos ejercen de intermediarios. En la "Red de Intermediación" de la Figura 3 se observa cómo tanto el Ayuntamiento de Málaga como la Junta de Andalucía se sitúan hacia el centro de la red. Hay un hecho diferencial con respecto a la red de intensidad y a la red de centralidad, puesto que la UMA y la CEM, que anteriormente se localizaban próximos al Ayuntamiento de Málaga y a la Junta de Andalucía, ahora se alejan bastante 
de los mismos, consecuencia de su menor intermediación. El resto de los actores se ubican en los extremos de la red al tener poca o ninguna capacidad de intermediar.

Figura 3. Red de Intermediación

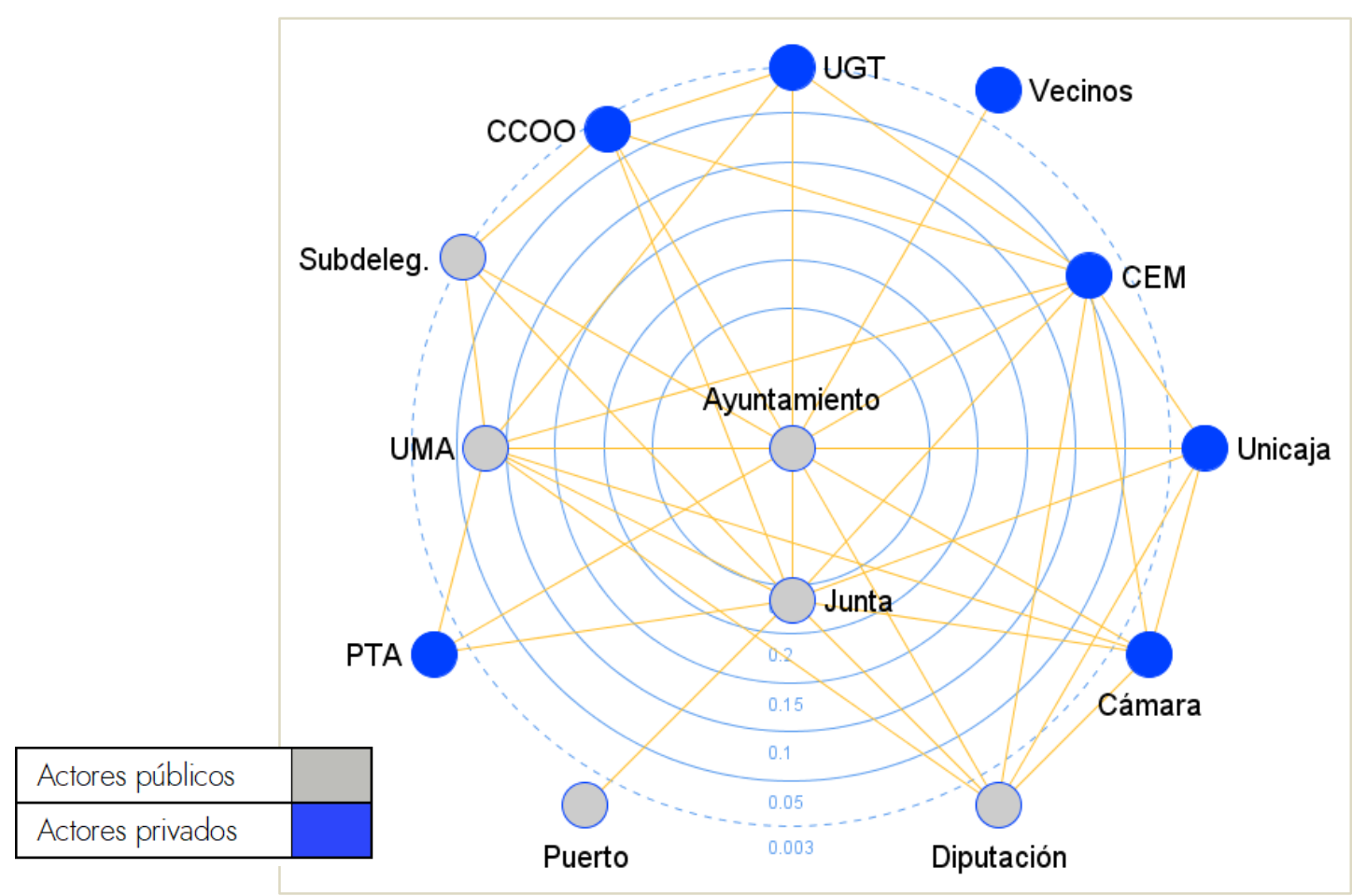

Fuente: elaboración propia con VISONE

\section{c) Grado de Cercanía}

El Grado de Cercanía muestra al Ayuntamiento de Málaga (92 308), la Junta de Andalucía (85 714), la UMA (80 000) y la CEM (75 000) como los patronos con más capacidad de conectarse con el resto de los patronos de la red. La Diputación Provincial, la Cámara de Comercio y Unicaja tienen la misma capacidad de llegar a los otros patronos (66 667), mientras que el sindicato CCOO (63 158) es el octavo patrono en cuanto a cercanía. Comparando la centralidad y la cercanía para estos patronos, se observa que su posición jerárquica, en ambos casos, en la misma. No obstante, existe un dato diferencial que desvela el grado de cercanía y es que UGT (57 143) y la Subdelegación del Gobierno (60 000), que tenían idénticos valores en centralidad y en intermediación, aquí se diferencian, siendo el segundo patrono el que mejor conexión tiene de los dos. El PTA iguala en capacidad de conexión a UGT, mientras que los dos patronos con peor conexión son la Federación de Asociaciones de Vecinos y la Autoridad Portuaria. La "Red de Cercanía" de la Figura 4 muestra cómo es la estructura reticular de este indicador. Aunque puede parecer en un primer acercamiento semejante a la red de centralidad en cuanto a posicionamiento de los patronos, estos, a excepción del Ayuntamiento de Málaga, están algo más alejados del centro de la red. 
Tabla 7. Grado de Cercanía

\begin{tabular}{|c|l|c|c|}
\hline \multicolumn{2}{|l|}{ Patronos } & Distancia & Cercanía \\
\hline 1 & Ayuntamiento de Málaga & 13000 & 92308 \\
\hline 2 & Junta de Andalucía & 14000 & 85714 \\
\hline 3 & UMA & 15000 & 80000 \\
\hline 4 & CEM & 16000 & 75000 \\
\hline 5 & Diputación Provincial & 18000 & 66667 \\
\hline- & Cámara de Comercio & 18000 & 66667 \\
\hline- & Unicaja & 18000 & 66667 \\
\hline 8 & CCOO & 19000 & 63158 \\
\hline 9 & $\begin{array}{l}\text { Subdelegación del } \\
\text { Gobierno }\end{array}$ & 20000 & 60000 \\
\hline 10 & UGT & 21000 & 57143 \\
\hline- & PTA & 21000 & 57143 \\
\hline 12 & Vecinos & 24000 & 50000 \\
\hline 13 & Autoridad Portuaria & 25000 & 48000 \\
\hline
\end{tabular}

Fuente: elaboración propia con UCINET 6

Figura 4. Red de Cercanía

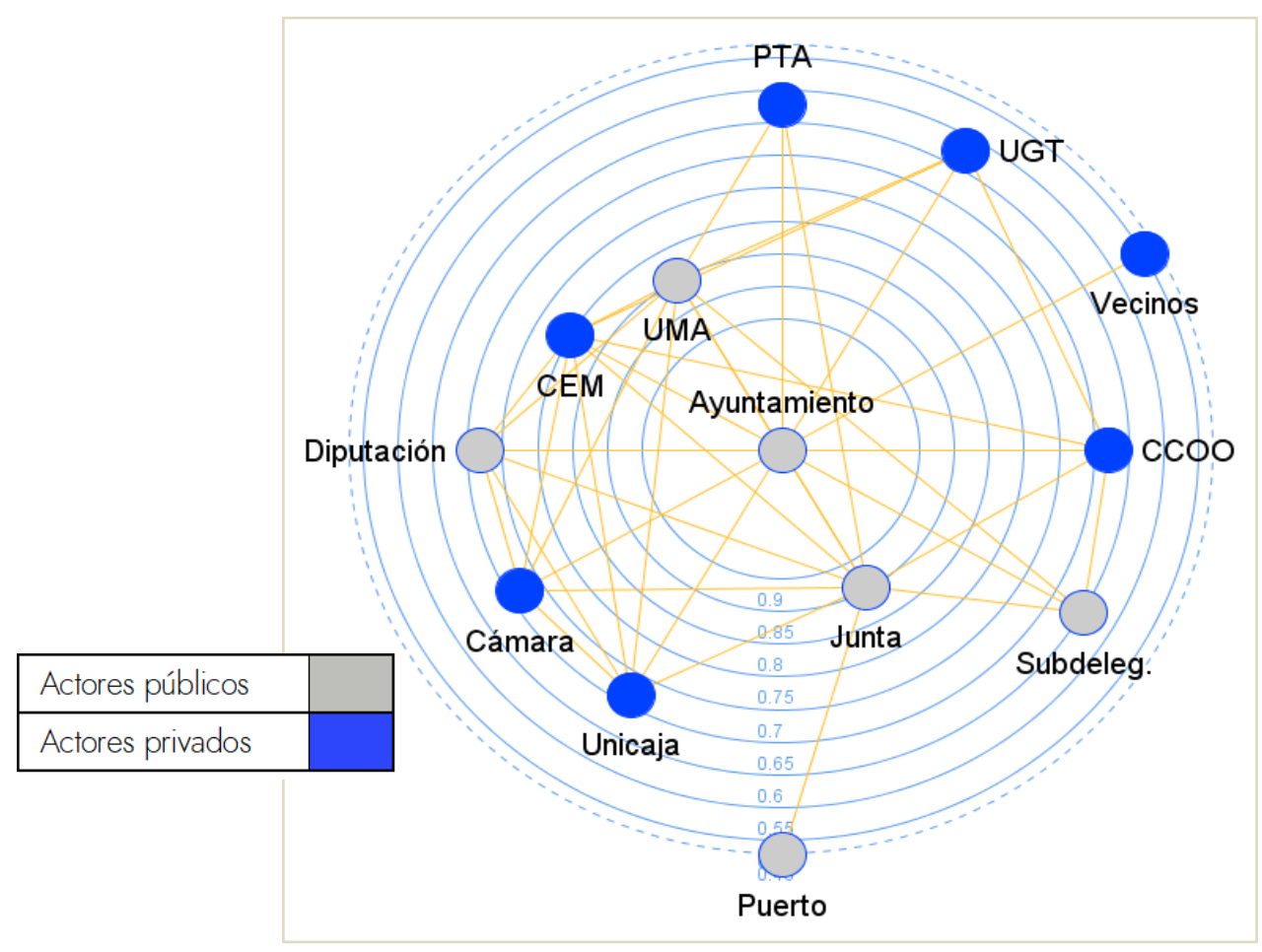

Fuente: elaboración propia con VISONE

d) Poder de Bonacich

El último indicador utilizado para analizar la red de gobernanza del Segundo Plan Estratégico de Málaga es el Poder de Bonacich. Bonacich (1987) diferencia entre centralidad y poder, señalando que los actores más centrales no son siempre los actores más poderosos. Así, un actor " $A$ " que está bien conectado a otros actores, que a su vez están bien conectados, hacen más central a " $A$ ", pero no poderoso. Por el contrario, si el actor " $A$ " está conectado a otros actores que no están 
bien conectados, hace que el primero sea más poderoso, porque estos últimos actores dependen del mismo para poder conectarse a la red. El Poder de Bonacich determina que la centralidad de un actor está en función de la cantidad de conexiones directas que éste tiene y cuántas conexiones tienen los actores a los que está conectado. En este caso ha interesado conocer las conexiones de los patronos a los que uno está conectado, por lo que se le ha considerado positivo el valor de beta $(\beta)^{1}{ }^{1}$ De esta manera, se ha determinado que la centralidad de un patrono está en función de la cantidad de conexiones directas que éste tiene y cuántas conexiones tienen los actores a los que está conectado.

Cuestiones que destacar de este indicador con respecto al grado de centralidad es que, para el Ayuntamiento de Málaga, la Junta de Andalucía, la UMA, la CEM, la Diputación Provincial, la Cámara de Comercio, Unicaja y CCOO las posiciones no cambian, por lo que además de ser centrales en cuanto a sus relaciones directas, lo son también en cuanto a su área de influencia o relaciones indirectas. Sin embargo, se observa un cambio para la Subdelegación del Gobierno y UGT, porque mientras que en el grado de centralidad ambos tenían el mismo dato, para el Poder de Bonacich difieren, con 0.484 y 0.474 respectivamente, lo que significa que el primer patrono tiene más capacidad de acceder a otros patronos, lo que lo convierte en más central. También hay diferencias entre la Federación de Asociaciones de Vecinos y la Autoridad Portuaria, porque mientras su grado de centralidad es el mismo, ahora el primero registra un mejor Poder de Bonacich, 0.143 frente 0.134 .

Tabla 8. Poder de Bonacich

\begin{tabular}{|c|l|c|c|}
\hline \multicolumn{2}{|l|}{ Patronos } & Valor Beta & Normalizada \\
\hline 1 & Ayuntamiento de Málaga & 3639961.250 & 1.000 \\
\hline 2 & Junta de Andalucía & 3420300.250 & 0.940 \\
\hline 3 & UMA & 3330402.250 & 0.915 \\
\hline 4 & CEM & 3169538.250 & 0.871 \\
\hline 5 & Diputación Provincial & 2709148.250 & 0.744 \\
\hline- & Cámara de Comercio & 2709148.250 & 0.744 \\
\hline- & Unicaja & 2709148.250 & 0.744 \\
\hline 8 & CCOO & 1958464.000 & 0.538 \\
\hline 9 & Subdelegación del Gobierno & 1762803.125 & 0.484 \\
\hline 10 & UGT & 1727014.750 & 0.474 \\
\hline 11 & PTA & 1483232.625 & 0.407 \\
\hline 12 & Vecinos & 519590.844 & 0.143 \\
\hline 13 & Autoridad Portuaria & 488238.281 & 0.134 \\
\hline
\end{tabular}

Fuente: elaboración propia con UCINET 6

1 Para un mayor desarrollo metodológico del Poder de Bonacich, ver Bonacich (1987), Rodríguez (2005) y Wasserman y Faust (2013). 


\section{Relación entre gobernanza y grado de ejecución de las líneas estratégicas}

En este apartado se analiza, de forma pormenorizada, la relación entre la gobernanza y el grado de ejecución de las líneas estratégicas. Para ello, se ha comparado la tendencia de los indicadores de resultado de las líneas estratégicas (grado de ejecución), la valoración sobre el grado de ejecución de las líneas estratégicas realizada por las partes interesadas y el número de coordinaciones ocurridas en cada una de las mismas. El grado de ejecución de cada línea estratégica se ha obtenido teniendo en cuenta, por un lado, el total de indicadores propuestos por la Fundación CIEDES y por otro, viendo cuáles de los mismos han tenido una tendencia positiva. ${ }^{2}$ Al ponderar ambos datos al valor 1, se obtiene un promedio de ejecución, expuesto en la Tabla 9. De esta manera, se puede observar que la línea estratégica con mayor grado de ejecución es "Málaga, ciudad litoral", con un promedio del 0.60, seguida de "Málaga, ciudad de la cultura" y "Málaga, ciudad renovada" con 0.52 y, finalmente, "Málaga, ciudad del conocimiento", con un promedio de 0.50 .

Asimismo, la valoración del grado de ejecución de las líneas estratégicas por las partes interesadas fue calculada por la Fundación CIEDES a través de un cuestionario que realizó a diferentes colectivos implicados y/o afectados por el plan estratégico. Dichos colectivos se clasificaron en técnicos, ciudadanos (periodistas, universitarios y ciudadanos) y patronos. Para obtener el dato de valoración de los colectivos e igualmente, el promedio general de la línea estratégica, se ponderaron los resultados al valor 5. De esta manera, como muestra la Tabla 10, la línea estratégica con mejor promedio de valoración es "Málaga, ciudad de la cultura" (3.51), seguida de "Málaga, ciudad litoral" (3.47), "Málaga, ciudad del conocimiento" (3.25) y "Málaga, ciudad renovada" (3.01). El colectivo de técnicos de la administración es el que mejor valora el grado de ejecución en todas las líneas estratégicas, mientras que los propios patronos de la Fundación CIEDES son los más críticos con la implementación de plan estratégico, al ser el colectivo que peor puntuación asigna al grado de ejecución de las líneas estratégicas, a excepción de la línea 2, que tiene la peor puntuación por parte de los ciudadanos.

Tabla 9. Grado de ejecución de las líneas estratégicas

\begin{tabular}{|l|c|c|c|}
\hline Líneas estratégicas & Total de indicadores & Tendencia positiva & Grado de ejecución \\
\hline Ciudad litoral & 15 & 9 & 0.60 \\
\hline Ciudad de la cultura & 67 & 35 & 0.52 \\
\hline Ciudad del conocimiento & 76 & 38 & 0.50 \\
\hline Ciudad renovada & 59 & 31 & 0.52 \\
\hline
\end{tabular}

Fuente: elaboración propia a partir de la Fundación CIEDES (2015)

2 En Fundación CIEDES (2015, pp. 200-209) se puede ver el listado total de indicadores y la tendencia de estos. 
Tabla 10. Valoración del grado de ejecución por las partes interesadas

\begin{tabular}{|l|c|c|c|c|}
\hline Líneas estratégicas & Técnicos & Ciudadanos & Patronos & Promedio \\
\hline Ciudad litoral & 3.68 & 3.49 & 3.26 & 3.47 \\
\hline Ciudad de la cultura & 3.71 & 3.37 & 3.45 & 3.51 \\
\hline Ciudad del conocimiento & 3.53 & 3.16 & 3.07 & 3.25 \\
\hline Ciudad renovada & 3.20 & 2.97 & 2.87 & 3.01 \\
\hline
\end{tabular}

Fuente: elaboración propia a partir de la Fundación CIEDES (2015)

Para conseguir el número de coordinaciones entre patronos por cada línea estratégica, se han contabilizado todas las actuaciones en donde al menos dos patronos se han coordinado en el diseño o en la implementación de los proyectos estrella y, consecuentemente, de las líneas estratégicas. En la Tabla 11 se refleja el resultado de estas coordinaciones, que, a diferencia de las matrices utilizadas anteriormente, que eran cuadradas, es decir, con la misma cantidad de filas que de columnas, ésta es de modo 2. Asimismo, es una matriz ponderada o valorada, al completarse con distintas cifras en función de la mayor o menor cantidad de coordinaciones entre los patronos por línea estratégica. Dicha cantidad de coordinaciones permite crear unos rangos de valores y así, obtener un dato de intensidad relacional. La clasificación que se ha establecido permite identificar coordinaciones intensas (16 o más), fluidas (entre 10 y 15), moderadas (entre 7 y 9), débiles (entre 4 y 6) y esporádicas (entre 1 y 3).

Tabla 11. Matriz de intensidad de coordinaciones entre patronos por línea estratégica

\begin{tabular}{|l|c|c|c|c|}
\cline { 2 - 5 } \multicolumn{1}{c|}{} & Ciudad litoral & Ciudad de la cultura & Ciudad del conocimiento & Ciudad renovada \\
\hline Ayto. & 6 & 15 & 13 & 21 \\
\hline Puerto & 4 & 0 & 0 & 3 \\
\hline Cámara & 1 & 3 & 7 & 5 \\
\hline CEM & 0 & 6 & 10 & 5 \\
\hline CCOO & 0 & 4 & 6 & 6 \\
\hline Junta & 6 & 11 & 21 & 4 \\
\hline Diputación & 1 & 4 & 4 & 4 \\
\hline Vecinos & 0 & 1 & 7 & 15 \\
\hline Subdeleg. & 1 & 4 & 6 & 6 \\
\hline PTA & 0 & 0 & 9 & 7 \\
\hline Unicaja & 0 & 5 & 21 & 5 \\
\hline UMA & 0 & 4 & 6 & 6 \\
\hline UGT & 0 & 3 & & 7 \\
\hline
\end{tabular}

\begin{tabular}{|l|l|}
\hline Intensa & 16 o más \\
\hline Fluida & $10-15$ \\
\hline Moderada & $7-9$ \\
\hline Débil & $4-6$ \\
\hline Esporádica & $1-3$ \\
\hline
\end{tabular}

Fuente: elaboración propia a partir de base de datos de la Fundación CIEDES

El grafo que resulta de la "Matriz de intensidad de coordinaciones entre patronos por línea estratégica" es la red de afiliación de la Figura 5. Este grafo permite una doble lectura, ya que, por 
un lado, posibilita ver en qué líneas estratégicas se producen las mayores coordinaciones y por otro, visibiliza cuáles son los patronos que más se coordinan y en qué líneas estratégicas lo hacen. Debido a que los valores en la matriz van desde el cero (ausencia de colaboración) hasta el 22 (Junta de Andalucía en "Málaga, ciudad renovada"), se han considerado los rangos de valores, es decir, la intensidad relacional, a la hora de construir el grafo. De esta forma, se consigue distinguir más claramente las coordinaciones, puesto que por un lado se reduce el número de enlaces y por otro, permite establecer niveles de colores y de grosor en los mismos. El máximo número de enlaces que puede recibir una línea estratégica es trece (uno por patrono), mientras que la intensidad relacional, como se ha comentado, es variable, dependiendo del número de ocasiones en que los patronos se coordinan en una determinada línea estratégica.

\section{Figura 5. Red de intensidad de coordinación entre patronos por línea estratégica}

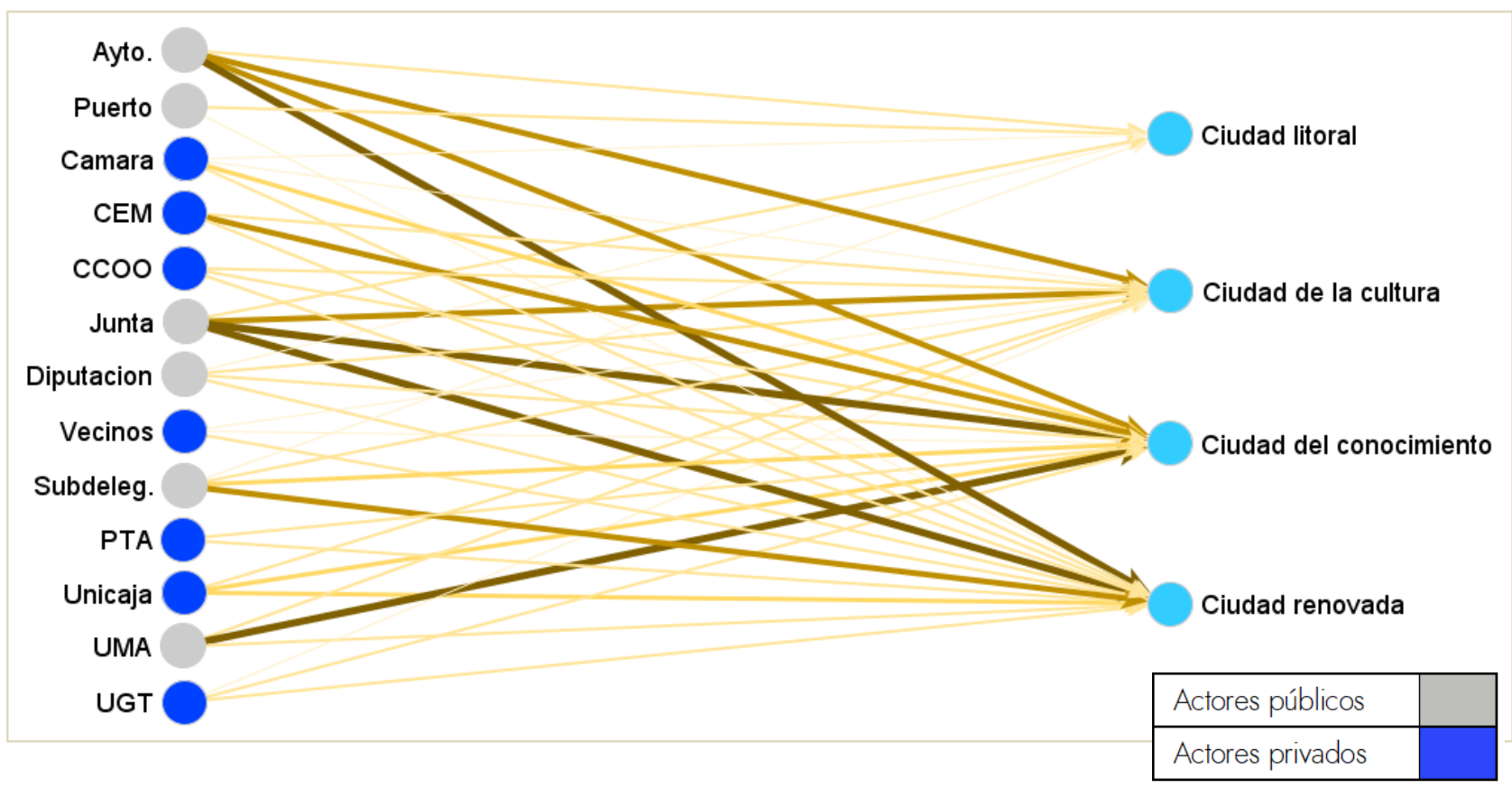

Fuente: elaboración propia con VISONE

Considerando lo anterior, la red de afiliación muestra que la línea estratégica 1, "Málaga, ciudad litoral", es la que menos coordinaciones recibe, tanto desde el punto de vista de la cantidad de enlaces como desde la óptica de la intensidad relacional. La coordinación en esta línea estratégica tiene un carácter predominantemente público, con poca implicación de los actores privados, ya que cinco de los seis patronos que han participado coordinándose para diseñar o implementar alguna actuación son públicos (Ayuntamiento de Málaga, Diputación Provincial, Junta de Andalucía, Subdelegación del Gobierno y Autoridad Portuaria). El grafo muestra, asimismo, que la coordinación en esta primera línea estratégica es las más baja de las cuatro, caracterizándose como débiles y esporádicas. No obstante, teniendo en cuenta el grado de ejecución, "Málaga, ciudad 
litoral" es la línea estratégica que mejor promedio tiene con 0.6, ya que, de los 15 indicadores considerados para medir esta línea, 9 tienen una tendencia positiva.

Por otro lado, excepto la Autoridad Portuaria y el PTA, el resto de los patronos se han coordinado, al menos una vez, en la línea estratégica 2, "Málaga, ciudad de la cultura", lo que significa que recibe once enlaces de trece posibles. Además de que hay más patronos que se coordinan en esta línea estratégica, la intensidad relacional también aumenta, destacando el Ayuntamiento de Málaga y la Junta de Andalucía con coordinaciones fluidas, con 15 y 11 respectivamente. En esta segunda línea estratégica, a diferencia de lo que ocurre en la primera, los actores privados tienen un papel relativamente importante, puesto que a pesar de que sus coordinaciones son débiles y esporádicas, todos, a excepción del PTA, han participado en alguna actuación. En lo que se refiere al grado de ejecución de "Málaga, ciudad de la cultura", es de 0.52, puesto que 35 indicadores tienen una tendencia positiva de los 67 utilizados.

En la línea estratégica 3, "Málaga, ciudad del conocimiento", es donde se producen las mayores coordinaciones entre patronos. A pesar de que la Autoridad Portuaria no ha participado en ninguna actuación en esta línea, lo que hace que reciba doce enlaces de trece posibles, la cantidad de coordinaciones entre patronos en esta línea estratégica es la mayor de las cuatro, destacando las coordinaciones intensas, fluidas y moderadas. En la red de afiliación se observa que la Junta de Andalucía y la UMA son los patronos principales en esta línea estratégica, al tener, ambos, coordinaciones de carácter intenso para con la misma. El Ayuntamiento de Málaga y la CEM se coordinan de una manera fluida, mientras que la Subdelegación del Gobierno, la Cámara de Comercio y la entidad bancaria Unicaja lo hacen de forma moderada. En esta tercera línea estratégica, los actores privados tienen un papel más destacado, el mayor de todas las líneas estratégicas del plan estratégico. El grado de ejecución de "Málaga, ciudad del conocimiento" es de 0.50, el más bajo de las cuatro líneas estratégicas, puesto que, de los 76 indicadores utilizados para medir esta línea, la mitad tiene una tendencia positiva.

En cuanto a la línea estratégica, "Málaga, ciudad renovada", es la única que recibe el máximo de enlaces posibles (trece de trece), aunque en términos de intensidad relacional tiene algo menos que la línea estratégica 3. Nuevamente, el Ayuntamiento de Málaga y la Junta de Andalucía son los patronos principales, aumentando su importancia, al tener, ambos, coordinaciones intensas. La Subdelegación del Gobierno se coordina, ahora, de una manera fluida, adquiriendo un protagonismo que en otras líneas no tiene. Asimismo, Unicaja destaca como el único patrono con coordinaciones moderadas. El resto de los patronos se coordinan de forma débil y sólo la participación de la Autoridad Portuaria es esporádica. En cuanto al grado de ejecución de "Málaga, ciudad renovada", este es de 0.52, el mismo promedio que la línea estratégica 2, puesto que, de los 59 indicadores utilizados para medir esta línea, 31 tienen una tendencia positiva. 
Finalmente, tras lo analizado, se puede concluir que la mayor coordinación entre los actores implicados en la implementación del Segundo Plan Estratégico de Málaga se da en aquellas líneas estratégicas que presentan un menor nivel de ejecución. La línea estratégica "Málaga, ciudad litoral" tiene el mayor grado de ejecución (0.60) y al mismo tiempo, la menor cantidad de coordinaciones entre patronos (sólo seis de trece) y con la menor intensidad (coordinaciones esporádicas y débiles). Por otro lado, "Málaga, ciudad del conocimiento", que es la línea estratégica con mayor intensidad de coordinación entre patronos y en donde los actores privados han tenido una importancia más significativa, es la que menor grado de ejecución tiene (0.50). Las líneas estratégicas "Málaga, ciudad de la cultura" y "Málaga, ciudad renovada", ambas con el Ayuntamiento de Málaga y la Junta de Andalucía como actores con más coordinaciones, tienen un grado de ejecución de 0.52, lo que significa que poco más de la mitad de las actuaciones que forman las mismas tienen una tendencia positiva. No obstante, tanto los patronos que participan como la intensidad de coordinación son distintas en ambas líneas, ya que en "Málaga, ciudad de la cultura" no todos los patronos participan y además no hay coordinaciones intensas, mientras que en "Málaga, ciudad renovada", todos los patronos participan y hay coordinaciones intensas, fluidas y moderadas.

Y esta misma tendencia se produce cuando se compara la valoración del grado de ejecución de las líneas estratégicas realizada por las partes interesadas y las relaciones de coordinación que se producen entre los patronos del plan estratégico. De esta forma, se puede comprobar que "Málaga, ciudad litoral" y "Málaga, ciudad de la cultura" tienen una mayor valoración en el grado de ejecución, 3.47 y 3.51 respectivamente, y, sin embargo, son las líneas estratégicas que presentan la menor intensidad relacional y la menor extensión de la colaboración. Lo contrario ocurre en "Málaga, ciudad del conocimiento" y "Málaga, ciudad renovada", que, siendo las líneas estratégicas con menor valoración del grado de ejecución, 3.25 y 3.01 respectivamente, son las que presentan mayor intensidad relacional y mayor extensión de la colaboración.

\section{Conclusiones}

Este trabajo se ha realizado desde la perspectiva de gobernanza, entendida como una forma de gobierno que tiene como fin dar respuesta a la complejidad propia de la resolución de problemas públicos que requieren de la implicación y participación de un amplio número de actores públicos y privados. Desde este punto de vista, la gobernanza se materializa en el concepto de red, y sus características, conocimiento y comprensión, son clave tanto para caracterizar a la propia forma de gobierno como para descubrir los patrones de interacción y poder incidir en la mejora de las decisiones públicas. Asimismo, el enfoque teórico-metodológico para analizar la gobernanza se ha centrado en la aplicación del ARS, poniendo el interés del análisis en las propiedades reticulares de la red a través de los distintos indicadores de densidad y de centralidad. 
Así, desde el punto de vista de las características del sistema de gobernanza aplicado en el Segundo Plan Estratégico de Málaga y en función de las propiedades reticulares, se destaca lo siguiente: en primer lugar, la densidad de la red de gobernanza es alta, es decir, son muchas las relaciones que se llevan a cabo entre los patronos para el impulso y realización de los proyectos del plan estratégico. Esto queda justificado con el indicador de densidad, que muestra que, de cada 100 relaciones posibles entre los patronos, se han producido casi 65. Como se comentó anteriormente, se considera que esta red, al estar formada por actores públicos y privados que responden a ámbitos competenciales diferentes y que, individualmente, pueden tener objetivos distintos (y a veces contradictorios), tiene una alta conectividad, con relaciones fuertes. Así, desde el punto de vista de la densidad de la red se puede afirmar que el desarrollo e implementación de las actuaciones recogidas en el plan estratégico se han fundamentado en la idea de coordinación y cooperación administrativa e interinstitucional, base para la gobernanza del territorio; en segundo lugar, la red de gobernanza desde el punto de vista de la centralidad es pluricéntrica, es decir, existen varios patronos que ejercen como actores centrales. Concretamente, tres actores del ámbito público (Ayuntamiento de Málaga, Junta de Andalucía y Universidad de Málaga) son los actores "líderes", aunque se puede comprobar que existe un conjunto de actores, principalmente, del ámbito privado, que ocupan posiciones moderadas en términos de centralidad y, por tanto, tienen capacidad de implicarse en las colaboraciones necesarias para el desarrollo de proyectos conjuntos. Destaca la posición de la Subdelegación del Gobierno, pues, en principio, debería ser más central por la afectación que en términos de acciones debería de impulsar para garantizar la ejecución de los proyectos del plan; en tercer lugar, teniendo en cuenta la intermediación, la capacidad de impulsar interconexiones entre actores en el sistema de gobernanza del Segundo Plan Estratégico de Málaga es limitada, puesto que, por un lado, casi la mitad de los patronos no tienen capacidad de intermediación (Diputación Provincial, Cámara de Comercio, Unicaja, PTA, Federación de Asociaciones de Vecinos y la Autoridad Portuaria) y, por otro, la capacidad de impulso de la colaboración para el desarrollo de las actuaciones está demasiado concentrada en unos pocos patronos; y en cuarto lugar, desde el punto de vista de la conexión individualizada de los actores, es posible mantener que existen dos patronos que están en situación de aislamiento en el sistema de gobernanza (Federación de Asociaciones de Vecinos y Autoridad Portuaria) y otros cuatro (UGT, CCOO, PTA y Subdelegación del Gobierno) tienen riesgos de aislamiento. Al contrario, la capacidad de realizar acciones conjuntas está limitada a pocos actores, siendo cuatro (Ayuntamiento de Málaga, Junta de Andalucía, UMA, y CEM) los que estarían en posiciones estructurales adecuadas para articular cooperación.

De forma general, se puede concluir que el sistema de gobernanza del Segundo Plan Estratégico de Málaga presenta una fortaleza importante en términos de la densidad de relaciones y de la existencia de varios actores que lideran la colaboración y la cooperación para el desarrollo conjunto 
de actuaciones. Pero, también es posible mantener que presenta un riesgo de generar vacíos que podrían provocar, de acuerdo a la dependencia estructural de pocos actores, la desconexión, y, por tanto, la no colaboración para el desarrollo de los proyectos del plan estratégico. Asimismo, el sistema de gobernanza presenta la característica básica de incorporar a actores públicos y privados que se relacionan y comparten recursos con el objetivo de resolver problemas complejos y comunes, que están en igualdad de condiciones de incorporarse a la red, aunque no en igualdad de posiciones, formado una estructura reticular pluricéntrica. De esta manera, la horizontalidad de las relaciones entre los actores, la aportación común y el grado de igualdad se convierten en la esencia de las relaciones de gobernanza.

Se ha puesto de manifiesto que existe una relación clara entre el nivel de ejecución de las líneas estratégicas del Segundo Plan Estratégico de Málaga y las redes de colaboración entre los actores implicados en el desarrollo de las actuaciones de estas, en el sentido de que, a menor grado de ejecución, mayor es la intensidad y la extensión de la colaboración entre dichos actores. Esta vinculación se puede producir por el hecho de que, proyectos y actuaciones con alta complejidad requieran de más coordinación y cooperación entre actores para poder ser llevadas a cabo, lo que, a su vez, dificulte la ejecución de estas. Este hecho permitiría plantear que no existe una vinculación directa entre más y mejor gobernanza y mayor ejecución de los proyectos, sino que la complejidad de estos estaría condicionada, también, directamente, por las exigencias de la gobernanza.

Por último, cabe destacar el papel central que los actores públicos tienen en el plan estratégico, tendencia que se repite, en general, en la planificación estratégica urbana. Asimismo, se constata una implicación más activa de los empresarios, como actores privados, que estaría denotando una cierta inclinación de la planificación estratégica urbana hacia proyectos con impacto económico y, por tanto, de mayor interés directo para los empresarios. En este sentido, se puede observar el modelo de la triple hélice, es decir, la tendencia de colaboración entre la Administración, la Universidad y el sector empresarial en los procesos de planificación, que tiene un claro componente innovador para el desarrollo del territorio.

Agradecimientos: Este trabajo de investigación se ha podido llevar a cabo gracias a la extraordinaria labor que desarrolla la Fundación CIEDES, institución responsable del Plan Estratégico de Málaga, para sistematizar y analizar la implementación a través de un sistema de seguimiento y evaluación. En esta labor de evaluación se concreta la medición continua de la gobernanza que se lleva a cabo en el plan estratégico, pues son conscientes de que dicho modelo de gobierno es el sustento mismo del proceso de planificación. Se dispone de un importante volumen de información sobre las redes de colaboración que se desarrollan en el proceso de planificación y sobre los que aplicar las técnicas propias del ARS para el estudio de las redes de gobernanza. Asimismo, el 
trabajo se inscribe en los proyectos "Análisis de la sostenibilidad urbana como estrategia de regeneración del espacio público de las áreas turísticas del litoral", financiado por la Fundación CajaCanarias y "Crisis y reestructuración de los espacios turísticos del litoral español", financiado por el Ministerio de Economía y Competitividad y el Fondo Europeo de Desarrollo Regional.

Declaración responsable: Las/os autoras/es declaran que no existe ningún conflicto de interés en relación con la publicación de este artículo. Las tareas se han distribuido de la siguiente manera: Rafael Merinero se ha encargado de analizar y sistematizar la literatura científica para el marco teórico en cuanto a la planificación estratégica y la gobernanza, y Oswaldo Ledesma la literatura de Análisis de Redes Sociales. Respecto al caso de Málaga, Rafael Merinero identificó la documentación para la caracterización y Oswaldo Ledesma realizó la redacción. Respecto al modelo analítico Rafael Merinero estructuró los elementos de base del modelo analítico y Oswaldo Ledesma identificó las características de los indicadores seleccionados. Sobre la redacción de los resultados tanto Rafael Merinero como Oswaldo Ledesma realizaron los análisis y redactaron los resultados. Las conclusiones fueron redactadas por Rafael Merinero y Oswaldo Ledesma. 


\section{Bibliografía}

Ansell, C., \& Gash, A. (2008). Collaborative governance in theory and practice. Journal of Public Administration Research and Theory, 18(4), 543-71. https://doi.org/10.1093/jopart/mum032

Barton, J. (2006). Sustentabilidad urbana como planificación estratégica. EURE-Revista Latinoamericana de Estudios Urbano Regionales, 32(96), 2746. http://dx.doi.org/10.4067/S0250-71612006000200003

Barzelay, M. (2007). Learning from Second-Hand Experience: Methodology for ExtrapolationOriented Case Research. Governance, 20(3), 521-543. https://doi.org/10.1111/j.1468$\underline{0491.2007 .00369 . x}$

Blanco, I. (2013). Analysing urban governance networks: Bringing regime theory back in. Environment and Planning C: Government and Policy, 31(2), 276291. https://doi.org/10.1068/c11174

Bonacich, P. (1987). Power and Centrality: A Family of Measures. American Journal of Sociology, 92(5), 1170-1182. https://doi.org/10.1086/228631

Borja, J., \& Castells, M. (1997). Local y Global. Madrid: Taurus.

Börzel, T. (2002). ¿ Qué tienen de especial los policy networks? Explorando el concepto y su utilidad para el estudio de la Gobernación europea. Retrieved from hittp://revistaredes.rediris.es/webredes/textos/policynet.pdf

Burt, R., \& Minor, M. (1983). Applied Network Analysis. Beverly Hills (California). Sage.

Busetti, S., \& Dente, B. (2018). Designing multi-actor implementation: A mechanism-based approach. Public Policy and Administration, 33(1), 4665. https://doi.org/10.1177/0952076716681207

Carrington, P., Scott, J., \& Wasserman, S. (Eds.) (2005). Models and Methods in Social Network Analysis. Nueva York. Cambridge University Press.

Chaques, L. (2004). Redes de políticas públicas. Madrid: Centro de Investigaciones Sociológicas.

Dente, B. (2007). Valutare il piano strategico o valutare il governo urbano? In T. Pugliese (Ed.), Monitoraggio e valutazione dei piani strategici (pp. 5-10). Florencia: Rete delle Città Strategiche.

Dente, B. (2011). Le decisioni di policy. Bolonia: II Mulino.

Dente, B., \& Coletti, P. (2011). Measuring Governance in Urban Innovation. Local Government Studies, 37(1), 43-56. https://doi.org/10.1080/03003930.2010.548553

Desouza, K., \& Flanery, T. (2013). Designing, planning, and managing resilient cities: A conceptual framework. Cities, 35, 89-99. https://doi.org/10.1016/j.cities.2013.06.003 
Elizalde, A. (2003). Planificación estratégica territorial y políticas públicas para el desarrollo local. Santiago de Chile. Instituto Latinoamericano y del Caribe de Planificación Económica y Social (ILPES), Naciones Unidas. Retrieved from https://repositorio.cepal.org/bitstream/handle/11362/7285/1/S03266_es.pdf

Falleti, T., \& Lynch, J. (2009). Context and Causal Mechanisms in Political Analysis. Comparative Political Studies, 20(10), 1-24. hittps://doi.org/10.1177/0010414009331724

Farinós, J. (2010). Nuevas prácticas de gobernanza para una renovada planificación territorial estratégica. Hacia la innovación socio-territorial. In A. Martín \& R. Merinero (Eds.), Metodología de la Planificación Estratégica Territorial (pp. 87-113). Sevilla: Junta de Andalucía.

Fernández-Güell, J.M. (2006). Planificación estratégica de ciudades: nuevos instrumentos y procesos. Barcelona: Reverté.

Fernández-Güell, J.M. (2007). 25 años de planificación estratégica de ciudades. Ciudad y Territorio, 39(154), 621-637.

Freeman, L. (2004). The development of social network analysis: a study in the sociology of science. Vancouver: Empirical Press.

Frewer, P. (2007). The need for new directions in metropolitan strategic planning. Urban Policiy And Research, 19(1), 93-100.

Fundación CIEDES (2006). II Plan Estratégico de Málaga. Málaga: Centro de Investigaciones Estratégicas y de Desarrollo Económico y Social (CIEDES). Retrieved from hittps://ciedes.es/images/stories/Libros_PEM/IIPEM.pdf

Fundación CIEDES (2010). Nuevos Rumbos. Reflexiones del Il Plan Estratégico de Málaga para el 2020. Málaga: Centro de Investigaciones Estratégicas y de Desarrollo Económico y Social (CIEDES). Retrieved from https://ciedes.es/images/stories/Libros_PEM/NuevosRumbos.pdf

Fundación CIEDES (2015). Evaluación del Il Plan Estratégico de Málaga 2007-2014 y propuesta marco para una Estrategia Integrada de Desarrollo Sostenible Málaga 2020. Málaga: Centro de Investigaciones Estratégicas y de Desarrollo Económico y Social (CIEDES). Retrieved from hitps://ciedes.es/images/stories/Libros_PEM/NuevosRumbos.pdf

Harrison, J., \& Hoyler, M. (2014). Governing the new metropolis. Urban Studies, 51(11), $2249-$ 2266. https://doi.org/10.1177/0042098013500699

Heinz, J., Laumann, E., Nelson, R., \& Salisbury, R. (1993). The Hollow Core: Private Interests in National Policy Making. Harvard: Harvard University Press. 
Howlett, M. (2002). Do Networks Matter? Linking Policy Network Structure to Policy Outcomes: Evidence from Four Canadian Policy Sectors 1990-2000. Canadian Journal of Political Science, 35(2), 235-267.

Jackson, M. (2010). Social and Economic Networks. Princeton New Jersey: Princeton University Press.

Jouve, B. (2005). Cuestiones sobre la gobernanza urbana (Estudis 19). Barcelona: Fundació Carles Pi i Sunyer.

Kemp, R. (1993). Strategic Planning for Local Government. A Handbook for Officials and Citizens. Jefferson. North Carolina: McFarland \& Company.

Kirst, M., Meister, G., \& Rowley, S. (1984). Policy Issue Networks: their influence on state policymaking. Policy Studies Journal, 13(2), 247-263. https://doi.org/10.1111/j.15410072.1984.tb00338.x

Klijn, E., \& Skelcher, C. (2007). Democracy and governance networks: compatible or not? Four conjectures and their implications for policy and practice. Public Administration, 85(3), 587608. https://doi.org/10.1111/j.1467-9299.2007.00662.x

Klijn, E., \& Koppenjan, J. (2012). Governance network theory: past, present and future. Policy and Politics, 40(4), 587-606. https://doi.org/10.1111/10.1332/030557312X655431

Knoke, D. (1990). Political Networks: The Structural Perspective. Cambridge: Cambridge University Press.

Knoke, D., \& Kuklinski, J. (1982). Network Analysis. London: Sage.

Knoke, D., \& Laumann, E. (1982). The Social Organization of National Policy Domains: An Exploration of Some Structural Hypotheses. In P. Marsden, \& N. Lin (Eds.), Social Structure and Network Analysis (pp. 255-270). Beverly Hills (California): Sage.

Krels, P. (2007). Planning Cities for the Future: The Successes and Failures of Urban Economic Strategies in Europe. Cheltenham (United Kingdom): Edward Edgar Publishing.

Laumann, E., \& Pappi, F. (1976). Networks of Collective Action: A Perspective on Community Influence Systems. New York: Academic Press.

Le Galès, P. (1998). Régulation, gouvernance et territoires. In J. Commaille \& B. Jobert (Dirs.), Lés metamorphoses de la régulation politique (pp. 203-240). Paris: LGDJ.

Le Galès, P. (2006). Le città europee. Società urbane, globalizzazione, governo locale. Bolonia: II Mulino. 
Mäntysalo, R., Jarenko, K., Nilsson, K., \& Saglie, I.-L. (2015). Legitimacy of Informal Strategic Urban Planning-Observations from Finland, Sweden and Norway. European Planning Studies, 23(2), 349-366. https://doi.org/10.1080/09654313.2013.861808

Marin, B., \& Mayntz, R. (1991). Policy Network: Empirical Evidence and Theoretical Considerations. Frankfurt: Campus Verlag.

Marsden, P., \& Lin, N. (1982). Social Structure and Network Analysis. Beverly Hills (California): Sage.

Marsh, D., \& Rhodes, R. (1992). Policy Networks in British Government. Oxford: Clarendon Press. Martí-Costa, M., \& Tomàs, M. (2016). Urban governance in Spain: From democratic transition to austerity policies. Urban Studies, 21072122. https://doi.org/10.1177/0042098016669452

Martín, A., \& Merinero, R. (Dir.) (2010). Planificación estratégica territorial. Estudios metodológicos. Sevilla: Junta de Andalucía, Consejería de Gobernación y Justicia.

Martinelli, F. (2005). La pianificazione strategica in Italia e in Europa. Metodologie ed esiti a confronto. Milano: Francoangeli.

Mayntz, R. (2001). El Estado y la sociedad civil en la gobernanza moderna. Revista del CLAD Reforma y Democracia, 21, 7-21.

Mayntz, R. (2002). Los Estados Nacionales y la gobernanza global. Revista CLAD Reforma y Democracia, 24, 29-44.

Meier, K., \& O’Toole, L. (2007). Modeling Public Management. Empirical analysis of the management-performance nexus. Public Management Review, 9(4), 503527. hittps://doi.org/10.1080/14719030701726630

Molina, J.L. (2001). El análisis de redes sociales. Una introducción. Barcelona: Ediciones Bellaterra. Newman, M. (2010). Networks: An Introduction. New York: Oxford University Press.

O'Toole, L. (2015). Networks and Networking: The Public Administrative Agendas. Public Administration Review, 75(3), 361-371. https://doi.org/10.1111/puar. 12281

O’Toole, L., \& Meier, K. (2004). Public Management in Intergovernmental Networks: Matching Structural Networks and Managerial Networking. Journal of Public Administration Research and Theory, 14(4), 469-494. hittps://doi.org/10.1093/jopart/muh032

Pappi, F., \& Knoke, D. (1991). Political Exchange in the German and American Labor Policy Domain. In M. Bernd \& M. Renate (Eds.), Policy Network. Empirical Evidence and Theoretical Considerations (pp. 179-208). Frankfurt: Campus Verlag. 
Pascual, J.M. (1999). La estrategia de las ciudades. Barcelona: Diputación de Barcelona.

Pascual, J.M., \& Tarragona, M. (2009). Estrategia Territorial y Gobierno Relacional. Manual para la Planificación Estratégica de $2^{a}$ Generación. Sevilla: Junta de Andalucía, Consejería de Gobernación.

Pinson, G. (2011). Urbanismo y gobernanza de las ciudades europeas. Gobernar la ciudad por proyecto. Valencia: Universidad de Valencia.

Requena, F. (2003). Análisis de redes sociales: orígenes, teorías y aplicaciones. Madrid: Centro de Investigaciones Sociológicas.

Rhodes, R. (1996). The New Governance: Governing without Government. Political Studies, 44, 652-667. https://doi.org/10.1111/j.1467-9248.1996.tb01747.x

Rhodes, R. (1997). Understanding Governance. Policy Networks, Governance, Reflexivity and Accountability. Buckingham: Open University Press.

Robins, G., Lewis, J., \& Wang, P. (2012). Statistical Network Analysis for Analyzing Policy Networks. Policy Studies Journal, 40(3), 375-401. https://doi.org/10.1111/j.1541$\underline{0072.2012 .00458 . x}$

Rodriguez, J. (2000). El círculo del poder: la estructura social del poder económico en la España de los noventa. Sistema, 158, 53-89.

Rodríguez, J. (2005). Análisis estructural y de redes. Madrid: Centro de Investigaciones Sociológicas.

Romero, J., \& Farinós, J. (2011). Redescubriendo la gobernanza más allá del buen gobierno. Democracia como base, desarrollo territorial como resultado. Boletín de la Asociación de Geógrafos Españoles, 56, 295-319.

Scott, J. (1991). Social Network Analysis: A Handbook. London: Sage.

Scott, J., \& Carrington, P. (Eds.) (2011). The Sage Handbook of Social Network Analysis. London: Sage.

Tanese, A., Di Filippo, E., \& Rennie, R. (2006). La pianificazione strategica per lo sviluppo dei territori. Rome: Rubbettino.

Tellería, I. (2014). Crisis de la gobernanza urbana y gestión de los comunes. Revista de Investigaciones Políticas y Sociológicas, 13(1), 33-47.

Tomàs M., \& Martí-Costa, M. (2011). La reconfiguración de la agenda urbana: el debate europeo. In M. Iglesias et al. (Eds.), Políticas urbanas en España. Grandes ciudades, actores y gobiernos locales (pp. 23-41). Barcelona: Icaria. 
Van Waarden, F. (1992). Dimensions and types of policy networks. European Journal of Political Research, 21, 29-52. https://doi.org/10.1111/j.1475-6765.1992.tb00287.x

Warsen, R., Nederhand, J., Klijn, E., Grotenberg, S., \& Koppenjan, J. (2018). What makes publicprivate partnerships work? Survey research into the outcomes and the quality of cooperation in PPPs. Public Management Review, 20(8), 11651185. https://doi.org/10.1080/14719037.2018.1428415

Wasserman, S., \& Galaskiewicz, J. (1994). Advances in Social Networks Analysis. Thousand Oaks (California): Sage.

Wasserman, S., \& Faust, K. (2013). Análisis de Redes Sociales. Métodos y Aplicaciones. Madrid: Centro de Investigaciones Sociológicas.

Wellman, B., \& Berkowitz, S. (Eds.) (1988). Social Structure: A Network Approach. Cambridge: Cambridge University Press. 